\title{
ON THE WARING-GOLDBACH PROBLEM FOR FOURTH AND FIFTH POWERS
}

\author{
KOICHI KAWADA and TREVOR D. WOOLEY
}

[Received 14 May 1999; revised 24 May 2000]

\section{Introduction}

Motivated by the general philosophy underlying applications of the HardyLittlewood method, it is conjectured that whenever $s$ and $k$ are positive integers with $s \geqslant k+1$, and $n$ is a large natural number satisfying the necessary local conditions, then $n$ should be represented as the sum of $s k$ th powers of prime numbers. In order to be somewhat explicit concerning the local conditions, suppose that $k$ is a natural number and $p$ is a prime number. We denote by $\theta=\theta(k, p)$ the integer with $p^{\theta} \mid k$ and $p^{\theta+1} \nmid k$, and then define $\gamma=\gamma(k, p)$ by

$$
\gamma(k, p)= \begin{cases}\theta+2, & \text { when } p=2 \text { and } \theta>0 \\ \theta+1, & \text { otherwise }\end{cases}
$$

Finally, we put

$$
K(k)=\prod_{(p-1) \mid k} p^{\gamma} .
$$

In particular, therefore, one has $K(4)=240$ and $K(5)=2$. Following Hua [8] (see, in particular, p. 108), we denote by $H(k)$ the least integer $s$ such that every sufficiently large positive integer congruent to $s$ modulo $K(k)$ may be written as a sum of $s k$ th powers of prime numbers. Note that when $(p-1) \mid k$ and $(p, a)=1$, one has $p^{\theta}(p-1) \mid k$, whence $a^{k} \equiv 1\left(\bmod p^{\gamma}\right)$. Thus it follows that whenever $n$ is the sum of $s k$ th powers of primes exceeding $k+1$, then necessarily $n$ is congruent to $s$ modulo $K(k)$. However, further congruence conditions may arise from primes $p$ with $(p-1) \nmid k$ (see, for example, Kawada [10]).

By the middle of this century, work of I. M. Vinogradov [20, 21], Hua (see [7] and [8]) and Davenport [4] had shown that

$$
\begin{aligned}
& H(1) \leqslant 3, \quad H(2) \leqslant 5, \quad H(3) \leqslant 9, \quad H(4) \leqslant 15, \\
& H(5) \leqslant 25, \quad H(6) \leqslant 37, \quad H(7) \leqslant 55, \quad H(8) \leqslant 75 .
\end{aligned}
$$

For $k \leqslant 4$, the above bounds for $H(k)$ remain the sharpest hitherto available. When $k \geqslant 5$, however, the new techniques developed in the mid-1980s by Thanigasalam

This paper was written while the first author visited the University of Michigan at Ann Arbor, and enjoyed the benefits of a Fellowship from the David and Lucile Packard Foundation.

Research of the second author was supported in part by NSF grant DMS-9622773 and a Fellowship from the David and Lucile Packard Foundation. This paper was completed during this author's stay at the Institute for Advanced Study and Princeton University, supported by the 1998 Salem Prize.

2000 Mathematics Subject Classification: 11P05, 11N36, 11L15, 11P55.

Proc. London Math. Soc. (3) 83 (2001) 1-50. (c) London Mathematical Society 2001. 
[11-14] and Vaughan [17] yield superior bounds. In particular, the former author has obtained the upper bounds

$$
H(5) \leqslant 23, \quad H(6) \leqslant 33, \quad H(7) \leqslant 47, \quad H(8) \leqslant 63 .
$$

The purpose of this paper is to establish new bounds for $H(k)$ when $k=4$ and 5 . We remark that the methods employed in the proof of these new bounds are somewhat novel in their use of estimates stemming directly from exponential sums over prime numbers in combination with the linear sieve, rather than the conventional methods which would rather 'waste' a variable or two by throwing minor arc estimates down to an auxiliary mean value estimate based on variables not restricted to be prime numbers. We express the hope that this more sophisticated treatment may inspire further work in this area.

THEOREM 1. One has $H(4) \leqslant 14$. In particular, every sufficiently large integer congruent to 14 modulo 240 may be written as the sum of 14 fourth powers of prime numbers.

The argument used to establish Theorem 1 may also be applied to establish the following conclusions, though we provide no details herein.

(i) For each positive number $A$, all but at most $O\left(N(\log N)^{-A}\right)$ of the integers $n \equiv 7(\bmod 240)$ with $1 \leqslant n \leqslant N$ are represented as the sum of 7 fourth powers of prime numbers.

(ii) One has $H(5) \leqslant 22$. In particular, every sufficiently large even integer is the sum of 22 fifth powers of prime numbers.

(iii) For each positive number $A$, all but at most $O\left(N(\log N)^{-A}\right)$ of the odd numbers $n$ with $1 \leqslant n \leqslant N$ are represented as the sum of 11 fifth powers of prime numbers.

We note that a formal application of the Hardy-Littlewood method leads one to expect that $H(4)=5$ and $H(5)=7$, based on a consideration of congruence conditions modulo 13 and 11 , respectively.

The method we apply to prove Theorem 1 narrowly fails to establish the sharper upper bound $H(5) \leqslant 21$. However, by appealing to Iwaniec's linear sieve in combination with the switching principle, we are able to surmount the difficulties associated with the proof of such a bound.

Theorem 2. One has $H(5) \leqslant 21$. In particular, every sufficiently large odd integer may be written as the sum of 21 fifth powers of prime numbers.

We establish Theorems 1 and 2 by means of the Hardy-Littlewood method, and this entails making use of various estimates for exponential sums. In $\S 2$ we discuss the Hardy-Littlewood dissection utilized in our subsequent argument, together with a number of estimates primarily of use in our analysis of the major arcs in the latter dissection. In order to provide appropriate upper bounds for exponential sums involving prime numbers, and allied sums pertinent to the sieving procedure described in $\S \S 7$ and 9 , it is necessary to consider estimates for bilinear and trilinear exponential sums. Such matters are discussed in $\S 3$. We derive mean value estimates for exponential sums over fourth powers from work of Vaughan [17] and Thanigasalam [14] in $\S 4$, and complete our proof of Theorem 1 
in $\S 5$ by making use of the technical estimates of $\S \S 2$ and 3 within the medium of the circle method.

The proof of Theorem 2, which we describe in $\S \S 6-9$, is complicated by our inability to consider directly sums of 21 fifth powers of prime numbers. Instead, we are forced to apply a sieving procedure in combination with the circle method to detect representations of a given integer $N$ in such a manner. A related device was employed by Heath-Brown [5] in the course of investigations concerning 4-term arithmetic progressions consisting of primes and almost-primes, and more recently in work of Brüdern $[\mathbf{1}, \mathbf{2}, \mathbf{3}]$, and Kawada [10] on the Waring-Goldbach problem. Thus, in $\S 6$ we establish the mean value estimates required in our application of the circle method for sums of fifth powers of primes. We aim to exclude almost-primes in order to detect only the representations of the desired integer $N$ as a sum of 21 fifth powers of prime numbers, and thus in $\S 7$ we estimate the contribution of the offensive almost-primes to the number of proposed representations. In order to achieve this end we employ a form of Iwaniec's linear sieve with the 'switching principle', such matters being deferred to $\S 9$. The proof of Theorem 2 is then completed on providing a comparison between the two singular series arising from our applications of the Hardy-Littlewood method, this being crucial to the sieving procedure.

As is usual, we abbreviate $e^{2 \pi i z}$ to $e(z)$, and write $[x]$ for the largest integer not exceeding $x$. The letter $p$, with or without a subscript, always denotes a prime number. We use $\varepsilon$ to denote a sufficiently small positive number, and take $P$ to be the main parameter, a real number sufficiently large in terms of $\varepsilon$ and $k$. We use $\ll$ and $\gg$ to denote Vinogradov's well-known notation, and write $A \asymp B$ as shorthand for the statement $A \ll B \ll A$. Implicit constants in the notations of Vinogradov and Landau may depend at most on $\varepsilon$ and $k$, unless otherwise indicated. Finally, we adopt the convention throughout that whenever $\varepsilon$ occurs in a statement, then the statement holds for each positive number $\varepsilon$.

\section{Preliminaries}

Before embarking on the substantive parts of the proofs of Theorems 1 and 2, we first present a number of technical estimates of which we make use in subsequent sections. When $k \geqslant 2$, we write

$$
S_{k}(q, a)=\sum_{r=1}^{q} e\left(a r^{k} / q\right)
$$

and define also the multiplicative function $w_{k}(q)$ by taking

$$
w_{k}\left(p^{u k+v}\right)= \begin{cases}k p^{-u-1 / 2}, & \text { when } u \geqslant 0 \text { and } v=1, \\ p^{-u-1}, & \text { when } u \geqslant 0 \text { and } 2 \leqslant v \leqslant k .\end{cases}
$$

Then according to Lemma 3 of [17], whenever $a \in \mathbb{Z}$ and $q \in \mathbb{N}$ satisfy $(a, q)=1$, one has

$$
q^{-1 / 2} \leqslant w_{k}(q) \ll q^{-1 / k},
$$

and

$$
q^{-1} S_{k}(q, a) \ll w_{k}(q) .
$$


It is convenient to combine the conclusions of Lemmata 2.4, 6.1 and 6.2 of Vaughan [19] in the shape of the following lemma.

Lemma 2.1. Let $k$ be a natural number with $k \geqslant 3$, let $X$ be a real number with $X \geqslant 2$, and suppose that $I$ is an interval contained in $[X, 2 X]$. Suppose also that $C_{1}$ and $C_{2}$ are fixed positive numbers. When $\alpha$ is a real number satisfying the condition that there exist $a \in \mathbb{Z}$ and $q \in \mathbb{N}$ with

$$
(a, q)=1, \quad 1 \leqslant q \leqslant C_{1} X^{k 2^{1-k}} \text { and }|q \alpha-a| \leqslant C_{2} X^{k\left(2^{1-k}-1\right)},
$$

one has

$$
\sum_{x \in I} e\left(\alpha x^{k}\right) \ll \frac{w_{k}(q) X}{1+X^{k}|\alpha-a / q|}
$$

and otherwise

$$
\sum_{x \in I} e\left(\alpha x^{k}\right) \ll X^{1-2^{1-k}+\varepsilon} .
$$

Similarly, when $\alpha$ satisfies the condition (2.4) one has

and otherwise

$$
\sum_{x \in I}(\log x) e\left(\alpha x^{k}\right) \ll \frac{w_{k}(q) X \log X}{1+X^{k}|\alpha-a / q|}
$$

$$
\sum_{x \in I}(\log x) e\left(\alpha x^{k}\right) \ll X^{1-2^{1-k}+\varepsilon}
$$

Proof. By Dirichlet's approximation theorem there exist $a \in \mathbb{Z}$ and $q \in \mathbb{N}$ with

$$
(a, q)=1, \quad 1 \leqslant q \leqslant 2^{k} k X^{k-1} \text { and }|q \alpha-a| \leqslant\left(2^{k} k X^{k-1}\right)^{-1} .
$$

When $q>X$, it follows from Weyl's inequality (see [19, Lemma 2.4]) that

$$
\sum_{x \in I} e\left(\alpha x^{k}\right) \ll X^{1-2^{1-k}+\varepsilon} .
$$

If $q \leqslant X$, meanwhile, we may combine Lemmata 6.1 and 6.2 of [19] with (2.3) to obtain

$$
\sum_{x \in I} e\left(\alpha x^{k}\right) \ll \frac{w_{k}(q) X}{1+X^{k}|\alpha-a / q|}+q^{1 / 2+\varepsilon} .
$$

But in circumstances where

$$
C_{1} X^{k 2^{1-k}}<q \leqslant X \text { or }|q \alpha-a|>C_{2} X^{k\left(2^{1-k}-1\right)},
$$

one deduces from (2.2) and (2.10) that

$$
\sum_{x \in I} e\left(\alpha x^{k}\right) \ll \frac{X}{\left(q+X^{k}|q \alpha-a|\right)^{1 / k}}+X^{1 / 2+\varepsilon} \ll X^{1-2^{1-k}} .
$$

On the other hand, when

$$
1 \leqslant q \leqslant C_{1} X^{k 2^{1-k}} \text { and }|q \alpha-a| \leqslant C_{2} X^{k\left(2^{1-k}-1\right)},
$$

then the first term on the right-hand side of (2.10) dominates the second term. 
Indeed, on recalling (2.2) we find that

$$
\frac{w_{k}(q) X}{1+X^{k}|\alpha-a / q|} \geqslant q^{1 / 2} \frac{X}{q+X^{k}|q \alpha-a|} \gg q^{1 / 2} X^{1-k 2^{1-k}},
$$

and the desired conclusion follows on noting that $k 2^{1-k}<1$ for $k \geqslant 3$. Thus we deduce that (2.5) holds when $\alpha$ satisfies the conditions (2.4), and from (2.9) and (2.11) we conclude that when $\alpha$ does not satisfy (2.4), then the estimate (2.6) holds.

The proof of the lemma is completed on observing that the estimates (2.7) and (2.8) follow immediately from (2.5) and (2.6) via partial summation.

Next we prepare a few results concerning $w_{k}(q)$. In this context, it is useful to write $v_{k}(q)$ for the multiplicative function of $q$ defined by taking

for $u \geqslant 0$ and $1 \leqslant v \leqslant k$.

$$
v_{k}\left(p^{u k+v}\right)=p^{u+1}
$$

Lemma 2.2. Let $k$ be a natural number with $k \geqslant 3$. Then for each $q \in \mathbb{N}$, one has

$$
\sum_{d \mid q} w_{k}(q / d) v_{k}(d)^{-1} \ll q^{\varepsilon} w_{k}(q) .
$$

Proof. Put

$$
A(q)=\sum_{d \mid q} w_{k}(q / d) v_{k}(d)^{-1}
$$

Since $A(q)$ is plainly a multiplicative function of $q$, the proof of the lemma will be completed by showing that for each prime $p$ and natural number $l$, one has

$$
A\left(p^{l}\right) \ll l w_{k}\left(p^{l}\right)
$$

But in view of the definition of $w_{k}(q)$, one has

and

$$
w_{k}\left(p^{l-k}\right)=p w_{k}\left(p^{l}\right) \text { for } l \geqslant k,
$$

$$
w_{k}\left(p^{l-h}\right) \leqslant k p w_{k}\left(p^{l}\right) \text { for } l \geqslant h \text { and } 0 \leqslant h \leqslant k-1 .
$$

Consequently, whenever $l \geqslant h \geqslant 0$, it follows that

$$
w_{k}\left(p^{l-h}\right) \leqslant k v_{k}\left(p^{h}\right) w_{k}\left(p^{l}\right)
$$

whence for $l \geqslant 1$ we arrive at the upper bound

$$
A\left(p^{l}\right)=\sum_{h=0}^{l} w_{k}\left(p^{l-h}\right) v_{k}\left(p^{h}\right)^{-1} \leqslant \sum_{h=0}^{l} k w_{k}\left(p^{l}\right) \ll l w_{k}\left(p^{l}\right),
$$

which is the desired estimate (2.12). This completes the proof of the lemma.

Lemma 2.3. Suppose that $N$ is a real number with $N \geqslant 2$, and $q \in \mathbb{N}$. Then

$$
\sum_{1 \leqslant n \leqslant N} w_{k}\left(q /\left(q, n^{k}\right)\right) n^{-1} \ll q^{\varepsilon} w_{k}(q) \log N .
$$


Proof. On recalling the definition of $v_{k}(q)$ and making use of multiplicativity, we find that $n^{k} \equiv 0(\bmod d)$ if and only if $n \equiv 0\left(\bmod v_{k}(d)\right)$. Consequently,

$$
\begin{aligned}
\sum_{1 \leqslant n \leqslant N} w_{k}\left(q /\left(q, n^{k}\right)\right) n^{-1} & \leqslant \sum_{d \mid q} w_{k}(q / d) \sum_{\substack{1 \leqslant n \leqslant N \\
n^{k} \equiv 0(\bmod d)}} n^{-1} \\
& \ll \sum_{d \mid q} w_{k}(q / d) v_{k}(d)^{-1} \log N .
\end{aligned}
$$

The proof of the lemma is completed on application of the conclusion of Lemma 2.2.

Lemma 2.4. Let $k$ be a natural number with $k \geqslant 3$, and suppose that $\eta$ and $\xi$ are real numbers satisfying $\eta>0, \xi>2 \eta+2$ and $\xi \geqslant k \eta+1$. Then whenever $Q \geqslant 2$, one has

$$
\sum_{1 \leqslant q \leqslant Q} q^{\eta} w_{k}(q)^{\xi} \ll \begin{cases}1, & \text { when } \xi>k \eta+1, \\ \log Q, & \text { when } \xi=k \eta+1,\end{cases}
$$

where the implied constant depends at most on $k, \eta$ and $\xi$.

Proof. In view of the multiplicative property of $w_{k}(q)$, one has

$$
\sum_{1 \leqslant q \leqslant Q} q^{\eta} w_{k}(q)^{\xi} \leqslant \prod_{p \leqslant Q}\left(1+\sum_{l=1}^{\infty} p^{\eta l} w_{k}\left(p^{l}\right)^{\xi}\right) .
$$

But from the definition of $w_{k}(q)$ we deduce that

$$
\sum_{l=1}^{\infty} p^{\eta l} w_{k}\left(p^{l}\right)^{\xi}=\sum_{u=0}^{\infty}\left(p^{\eta(k u+1)}\left(k p^{-u-1 / 2}\right)^{\xi}+\sum_{v=2}^{k} p^{\eta(k u+v)}\left(p^{-u-1}\right)^{\xi}\right),
$$

whence

$$
\begin{aligned}
& \sum_{l=1}^{\infty} p^{\eta l} w_{k}\left(p^{l}\right)^{\xi}-\sum_{u=0}^{\infty} p^{-(\xi-k \eta)(u+1)} \\
& \ll \sum_{u=0}^{\infty}\left(p^{-(\xi-k \eta) u-(\xi-2 \eta) / 2}+p^{-(\xi-k \eta)(u+1)-\eta}\right)
\end{aligned}
$$

Thus we obtain the estimate

$$
\sum_{l=1}^{\infty} p^{\eta l} w_{k}\left(p^{l}\right)^{\xi}-p^{-(\xi-k \eta)} \ll p^{-2(\xi-k \eta)}+p^{-(\xi-2 \eta) / 2}+p^{-(\xi-k \eta)-\eta} .
$$

In view of our hypotheses concerning $\xi$ and $\eta$, it follows that there is a positive number $\nu$ for which the right-hand side of (2.14) is $O\left(p^{-1-\nu}\right)$. Thus, when $\xi>k \eta+1$ one deduces from (2.13) that

$$
\sum_{1 \leqslant q \leqslant Q} q^{\eta} w_{k}(q)^{\xi} \leqslant \prod_{p \leqslant Q}\left(1+p^{-(\xi-k \eta)}+O\left(p^{-1-\nu}\right)\right) \ll 1,
$$

and when $\xi=k \eta+1$, a well-known prime number estimate reveals in like 
manner that

$$
\sum_{1 \leqslant q \leqslant Q} q^{\eta} w_{k}(q)^{\xi} \leqslant \prod_{p \leqslant Q}\left(1+p^{-1}+O\left(p^{-1-\nu}\right)\right) \ll \log Q .
$$

This completes the proof of the lemma.

We make use of various Farey dissections of the unit interval in the sequel, and so at this point it is convenient to fix a uniform notation. When $X>1$ and $Y>2 X^{2}$, we define the major $\operatorname{arcs} \mathfrak{M}(X ; Y)$ to be the union of the intervals

$$
\mathfrak{M}(q, a ; X ; Y)=\left\{\alpha \in[0,1):|q \alpha-a| \leqslant X Y^{-1}\right\},
$$

with $0 \leqslant a \leqslant q \leqslant X$ and $(a, q)=1$. We then define

$$
\mathfrak{m}(X ; Y)=[0,1) \backslash \mathfrak{M}(X ; Y) \text {. }
$$

Note that when $1 \leqslant q, q^{\prime} \leqslant X,(a, q)=\left(a^{\prime}, q^{\prime}\right)=1$ and $a / q \neq a^{\prime} / q^{\prime}$, then our hypotheses on $X$ and $Y$ imply that

$$
\left|\frac{a}{q}-\frac{a^{\prime}}{q^{\prime}}\right| \geqslant \frac{1}{q q^{\prime}}>\frac{2 X^{2}}{q q^{\prime} Y} \geqslant \frac{\left(q+q^{\prime}\right) X}{q q^{\prime} Y}=\frac{X}{q Y}+\frac{X}{q^{\prime} Y},
$$

whence the intervals $\mathfrak{M}(q, a ; X ; Y)$ comprising $\mathfrak{M}(X ; Y)$ are pairwise disjoint.

We close this section by establishing a variant of Lemma 4 of Brüdern [3].

Lemma 2.5. Let $k$ be a natural number with $k \geqslant 4$, and let $Q$ and $P$ be real numbers with $1 \leqslant Q \leqslant P$. Write

$$
g(\alpha)=\sum_{Q<p \leqslant 2 Q} e\left(p^{k} \alpha\right)
$$

and when $\eta$ is a positive number, let $\Psi_{\eta}(\alpha)$ be the function defined on $\mathfrak{M}\left(Q ; P^{k}\right)$ by taking

$$
\Psi_{\eta}(\alpha)=q^{\eta} w_{k}(q)\left(1+P^{k}|\alpha-a / q|\right)^{-1}
$$

for $\alpha \in \mathfrak{M}\left(q, a ; Q ; P^{k}\right) \subseteq \mathfrak{M}\left(Q ; P^{k}\right)$. Then whenever $0<\eta \leqslant(4 k)^{-1}$, one has

$$
\int_{\mathfrak{M}\left(Q ; P^{k}\right)} \Psi_{\eta}(\alpha)^{3}|g(\alpha)|^{2} d \alpha \ll Q^{2} P^{-k}
$$

Proof. We have

$$
\int_{\mathfrak{M}\left(Q ; P^{k}\right)} \Psi_{\eta}(\alpha)^{3}|g(\alpha)|^{2} d \alpha \ll \sum_{1 \leqslant q \leqslant Q} q^{3 \eta} w_{k}(q)^{3} \mathfrak{J}(Q, q),
$$

where

$$
\mathfrak{J}(Q, q)=\sum_{\substack{a=1 \\(a, q)=1}}^{q} \int_{0}^{Q P^{-k}}\left(1+P^{k} \beta\right)^{-3}|g(\beta+a / q)|^{2} d \beta .
$$


But

$$
\begin{aligned}
\sum_{\substack{a=1 \\
(a, q)=1}}^{q}|g(\beta+a / q)|^{2} & \leqslant \sum_{a=1}^{q}\left|\sum_{Q<p \leqslant 2 Q} e\left(p^{k}(\beta+a / q)\right)\right|^{2} \\
& \leqslant q \sum_{\substack{Q<p_{1}, p_{2} \leqslant 2 Q \\
p_{1}^{k} \equiv p_{2}^{k}(\bmod q)}} 1 .
\end{aligned}
$$

For each pair $p_{1}, p_{2}$ occurring in the latter summation, whenever $q \leqslant Q$ one plainly has $\left(q, p_{1} p_{2}\right)=1$. But when $(h, q)=1$, the number of solutions $x$ modulo $q$ of the congruence $x^{k} \equiv h(\bmod q)$ is $O\left(q^{\varepsilon}\right)$, and thus

$$
\sum_{\substack{a=1 \\(a, q)=1}}^{q}|g(\beta+a / q)|^{2} \ll q^{1+\varepsilon} Q\left(Q q^{-1}+1\right) \ll q^{\varepsilon} Q^{2} .
$$

We therefore deduce from (2.18) that

$$
\mathfrak{J}(Q, q) \ll q^{\varepsilon} Q^{2} \int_{0}^{\infty}\left(1+P^{k} \beta\right)^{-3} d \beta \ll q^{\varepsilon} Q^{2} P^{-k},
$$

whence by (2.17) and Lemma 2.4 it follows that

$$
\int_{\mathfrak{M}\left(Q ; P^{k}\right)} \Psi_{\eta}(\alpha)^{3}|g(\alpha)|^{2} d \alpha \ll Q^{2} P^{-k} \sum_{1 \leqslant q \leqslant Q} q^{1 / k} w_{k}(q)^{3} \ll Q^{2} P^{-k} .
$$

This completes the proof of the lemma.

\section{Weyl sums and multilinear exponential sums}

The purpose of this section is to provide estimates for both Weyl sums over prime numbers, and also certain multilinear exponential sums, of use in our application of the Hardy-Littlewood method. We begin by estimating a bilinear exponential sum. Here and throughout we write $\tau(m)$ for the divisor function.

Lemma 3.1. Let $k$ be a natural number with $k \geqslant 4$, and let $P, P^{\prime}, M, M^{\prime}, U$ and $U^{\prime}$ be positive real numbers with

$$
P^{1 / 2} \leqslant M \leqslant P, \quad P \leqslant P^{\prime} \leqslant 2 P \text { and } M \leqslant M^{\prime} \leqslant 2 M .
$$

Suppose that $\left(a_{m}\right)$ and $\left(b_{n}\right)$ are sequences of complex numbers satisfying the inequalities

$$
\left|a_{m}\right| \leqslant \tau(m)+\log m \text { and }\left|b_{n}\right| \leqslant \log n
$$

for each $m$ and $n$. Suppose further that $\alpha$ is a real number, and that there exist $a \in \mathbb{Z}$ and $q \in \mathbb{N}$ with

$$
(a, q)=1, \quad 1 \leqslant q \leqslant P^{k / 2} \text { and }|q \alpha-a| \leqslant P^{-k / 2} .
$$


Then one has

$$
\begin{aligned}
\sum_{M<m \leqslant M^{\prime}} a_{m} \sum_{\substack{P / m<n \leqslant P^{\prime} / m \\
U<n \leqslant U^{\prime}}} b_{n} e\left((m n)^{k} \alpha\right) \\
\ll P M^{\varepsilon-2^{-k}}+(P M)^{1 / 2+\varepsilon}+\frac{q^{\varepsilon} w_{k}(q)^{1 / 2} P(\log P)^{4}}{\left(1+P^{k}|\alpha-a / q|\right)^{1 / 2}} .
\end{aligned}
$$

Proof. Our proof is motivated by Vaughan's arguments appearing in [18, §3]. The first task is to exploit the bilinearity of the exponential sum through an application of Cauchy's inequality. Noting first that our hypotheses on $\left(a_{m}\right)$ ensure that

$$
\sum_{M<m \leqslant 2 M}\left|a_{m}\right|^{2} \ll M(\log M)^{3},
$$

an application of Cauchy's inequality reveals that

$$
\left|\sum_{M<m \leqslant M^{\prime}} a_{m} \sum_{\substack{P / m<n \leqslant P^{\prime} / m \\ U<n \leqslant U^{\prime}}} b_{n} e\left((m n)^{k} \alpha\right)\right|^{2} \ll M(\log M)^{3} S_{0},
$$

where

$$
S_{0}=\sum_{M<m \leqslant 2 M}\left|\sum_{\substack{P<n \leqslant P^{\prime} / m \\ U<n \leqslant U^{\prime}}} b_{n} e\left((m n)^{k} \alpha\right)\right|^{2} .
$$

Write $N=P /(2 M)$ and

$$
I\left(n_{1}, n_{2}\right)=(M, 2 M] \cap\left(P / \min \left\{n_{1}, n_{2}\right\}, P^{\prime} / \max \left\{n_{1}, n_{2}\right\}\right] .
$$

Then by expanding the square and interchanging the order of summation, we find that

$$
S_{0} \ll \sum_{N<n_{1}, n_{2} \leqslant 4 N}\left|b_{n_{1}} b_{n_{2}}\right|\left|\sum_{m \in I\left(n_{1}, n_{2}\right)} e\left(\left(n_{1}^{k}-n_{2}^{k}\right) m^{k} \alpha\right)\right| .
$$

Write

$$
S_{1}=\sum_{N<n_{1}<n_{2} \leqslant 4 N}\left|\sum_{m \in I\left(n_{1}, n_{2}\right)} e\left(\left(n_{2}^{k}-n_{1}^{k}\right) m^{k} \alpha\right)\right| .
$$

Then on recalling our hypotheses on $\left(b_{n}\right)$ and isolating the terms with $n_{1}=n_{2}$, we deduce that

$$
S_{0} \ll(\log P)^{2}\left(N M+S_{1}\right) \ll(\log P)^{2}\left(P+S_{1}\right) .
$$

Denote by $\mathscr{N}$ the set of ordered pairs $\left(n_{1}, n_{2}\right)$, with $N<n_{1}<n_{2} \leqslant 4 N$, for which there exist $b \in \mathbb{Z}$ and $r \in \mathbb{N}$ with

$$
\begin{aligned}
& (b, r)=1, \quad 1 \leqslant r \leqslant 2^{-2 k} M^{k 2^{1-k}}, \\
& \left|r\left(n_{2}^{k}-n_{1}^{k}\right) \alpha-b\right| \leqslant \frac{1}{2} M^{k\left(2^{1-k}-1\right)} .
\end{aligned}
$$

Then by Lemma 2.1 we have

$$
S_{1} \ll S_{2}+N^{2} M^{1-2^{1-k}+\varepsilon},
$$


where

$$
S_{2}=\sum_{\left(n_{1}, n_{2}\right) \in \mathcal{N}} \frac{w_{k}(r) M}{1+M^{k}\left|\left(n_{2}^{k}-n_{1}^{k}\right) \alpha-b / r\right|},
$$

and here $b$ and $r$ are the integers defined in (3.4) (which may, of course, depend on $n_{1}$ and $\left.n_{2}\right)$. When $\left(n_{1}, n_{2}\right) \in \mathscr{N}$, we put

$$
n_{0}=\left(n_{1}, n_{2}\right), \quad n=n_{1} / n_{0} \quad \text { and } \quad l=\left(n_{2}-n_{1}\right) / n_{0} \text {. }
$$

Define also $D=D(n, l)$ by

$$
D=\left((n+l)^{k}-n^{k}\right) / l,
$$

and note that $k n^{k-1}<D<k(4 N)^{k-1}$. Then on substituting these expressions into (3.6), we find that

$$
S_{2} \ll \sum_{1 \leqslant n_{0} \leqslant 4 N} \sum_{1 \leqslant l \leqslant 4 N / n_{0}} \sum_{\begin{array}{c}
N / n_{0}<n \leqslant 4 N / n_{0} \\
(n, l)=1 \\
\left(n n_{0},(n+l) n_{0}\right) \in \mathcal{N}
\end{array}} \frac{w_{k}(r) M}{1+M^{k}\left|n_{0}^{k} l D \alpha-b / r\right|} .
$$

For each pair $\left(n_{0}, l\right)$ occurring in the summations of (3.7), we apply Dirichlet's approximation theorem to deduce the existence of $c \in \mathbb{Z}$ and $s \in \mathbb{N}$ with

$$
(c, s)=1, \quad 1 \leqslant s \leqslant M^{k\left(1-2^{1-k}\right)} \text { and }\left|s n_{0}^{k} l \alpha-c\right| \leqslant M^{k\left(2^{1-k}-1\right)} .
$$

In view of (3.4), one has

$$
\begin{aligned}
|c r D-b s| & \leqslant r D M^{k\left(2^{1-k}-1\right)}+\frac{1}{2} s M^{k\left(2^{1-k}-1\right)} \\
& <\frac{1}{2}+2^{-2 k} k M^{k\left(2^{2-k}-1\right)}(4 N)^{k-1} .
\end{aligned}
$$

On recalling that $N=P /(2 M)$ and $M \geqslant P^{1 / 2}$, and noting that for $k \geqslant 4$ one has $k\left(2-2^{2-k}\right)-1 \geqslant 2(k-1)$, we therefore deduce that

$$
|c r D-b s|<\frac{1}{2}+k 2^{-k-1} P^{k-1} M^{1-k\left(2-2^{2-k}\right)}<1 .
$$

Thus we have

$$
\frac{b}{r D}=\frac{c}{s}, \quad r=\frac{s}{(s, D)},
$$

and, on writing $N_{0}=N / n_{0}$, we obtain

$$
\left|n_{0}^{k} l D \alpha-b / r\right|=D\left|n_{0}^{k} l \alpha-c / s\right| \gg N_{0}^{k-1}\left|n_{0}^{k} l \alpha-c / s\right| .
$$

Hence we conclude from (3.7) that

$$
\begin{aligned}
& S_{2} \ll \sum_{1 \leqslant n_{0} \leqslant 4 N} \sum_{1 \leqslant l \leqslant 4 N / n_{0}} \frac{M}{1+M^{k} N_{0}^{k-1}\left|n_{0}^{k} l \alpha-c / s\right|} \\
& \times \sum_{\substack{1 \leqslant n \leqslant 4 N / n_{0} \\
(n, l)=1}} w_{k}(s /(s, D)) .
\end{aligned}
$$

We now investigate the innermost sum in (3.8), starting from the inequality

$$
\sum_{\substack{1 \leqslant n \leqslant 4 N / n_{0} \\(n, l)=1}} w_{k}(s /(s, D)) \leqslant \sum_{s_{0} \mid s} w_{k}\left(s / s_{0}\right) \sum_{\substack{1 \leqslant n \leqslant 4 N / n_{0} \\(n, l)=1 \\ D \equiv 0\left(\bmod s_{0}\right)}} 1 .
$$


Note first that whenever $n$ occurs in the innermost sum of (3.9), then $(n, l)=1$ and

$$
D=\left((n+l)^{k}-n^{k}\right) / l \equiv 0 \quad\left(\bmod s_{0}\right),
$$

whence $\left(n, s_{0}\right)=1$. Write

$$
s_{1}=\left(l, s_{0}\right), \quad s_{2}=s_{0} / s_{1}, \quad l_{0}=l / s_{1},
$$

and let $\bar{n}$ be an integer satisfying the congruence $n \bar{n} \equiv 1\left(\bmod s_{0}\right)$. Then it follows from (3.10) that

$$
\left(1+l_{0} s_{1} \bar{n}\right)^{k} \equiv 1 \quad\left(\bmod s_{0}\right) .
$$

But the congruence $y^{k} \equiv 1\left(\bmod s_{0}\right)$ has at most $O\left(s_{0}^{\varepsilon}\right)$ solutions modulo $s_{0}$, say $y_{1}, \ldots, y_{\nu}$ for some integer $\nu$ with $1 \leqslant \nu \ll s_{0}^{\varepsilon}$. Then $1+l_{0} s_{1} \bar{n} \equiv y_{j}\left(\bmod s_{0}\right)$ for some $j$ with $1 \leqslant j \leqslant \nu$, and hence $y_{j} \equiv 1\left(\bmod s_{1}\right)$ and $l_{0} \bar{n} \equiv\left(y_{j}-1\right) / s_{1}\left(\bmod s_{2}\right)$. Since $\left(l_{0}, s_{2}\right)=1$, the latter congruence implies that $\bar{n}$ belongs to one of $O\left(s_{0}^{\varepsilon}\right)$ residue classes modulo $s_{2}$, and hence $n$ likewise belongs to one of $O\left(s_{0}^{\varepsilon}\right)$ residue classes modulo $s_{2}$. On the other hand, since $k n^{k-1} \equiv D \equiv 0\left(\bmod s_{1}\right)$ and $\left(n, s_{1}\right)=1$, one has $s_{1} \mid k$. Thus $s_{2}=s_{0} / s_{1} \gg s_{0}$, and so by (3.9) and (2.2),

$$
\begin{aligned}
\sum_{\substack{1 \leqslant n \leqslant 4 N / n_{0} \\
(n, l)=1}} w_{k}(s /(s, D)) & \ll \sum_{s_{0} \mid s} w_{k}\left(s / s_{0}\right) s_{0}^{\varepsilon}\left(N\left(n_{0} s_{0}\right)^{-1}+1\right) \\
& \ll s^{\varepsilon} N n_{0}^{-1} \sum_{s_{0} \mid s} s_{0}^{-1} w_{k}\left(s / s_{0}\right)+s^{\varepsilon} \sum_{s_{0} \mid s} 1 .
\end{aligned}
$$

Consequently, recalling that $v_{k}(d) \leqslant d$, we deduce from Lemma 2.2 that

$$
\sum_{\substack{1 \leqslant n \leqslant 4 N / n_{0} \\(n, l)=1}} w_{k}(s /(s, D)) \ll s^{\varepsilon} w_{k}(s) N n_{0}^{-1}+s^{\varepsilon} .
$$

Next write $P_{0}=P / n_{0}$ and

$$
T\left(n_{0}, l\right)=\frac{s^{\varepsilon} w_{k}(s) P n_{0}^{-1}}{1+P_{0}^{k-1} M\left|n_{0}^{k} l \alpha-c / s\right|} .
$$

Then on combining (3.8) and (3.11), we obtain

$$
\begin{aligned}
S_{2} & \ll \sum_{1 \leqslant n_{0} \leqslant 4 N} \sum_{1 \leqslant l \leqslant 4 N / n_{0}}\left(T\left(n_{0}, l\right)+s^{\varepsilon} M\right) \\
& \ll \sum_{1 \leqslant n_{0} \leqslant 4 N} \sum_{1 \leqslant l \leqslant 4 N / n_{0}} T\left(n_{0}, l\right)+P^{1+\varepsilon} .
\end{aligned}
$$

In view of (2.2), one has

$$
T\left(n_{0}, l\right) \ll \frac{s^{\varepsilon} P n_{0}^{-1}}{\left(s+P_{0}^{k-1} M\left|s n_{0}^{k} l \alpha-c\right|\right)^{1 / k}},
$$

whence

$$
T\left(n_{0}, l\right) \ll P M^{\varepsilon-2^{1-k}},
$$

except possibly when

$$
1 \leqslant s \leqslant M^{k 2^{1-k}} \text { and }\left|s n_{0}^{k} l \alpha-c\right| \leqslant \frac{1}{2} M^{k 2^{1-k}-1} P^{1-k} .
$$


For each integer $n_{0}$ satisfying $1 \leqslant n_{0} \leqslant 4 N$, we denote by $\mathscr{L}$ the set of natural numbers $l$ with $1 \leqslant l \leqslant 4 N / n_{0}$ for which the conditions (3.13) are satisfied. Then on writing

$$
S_{3}=\sum_{1 \leqslant n_{0} \leqslant 4 N} \sum_{l \in \mathscr{L}} T\left(n_{0}, l\right),
$$

we deduce from (3.5) and (3.12) that

$$
S_{1} \ll S_{3}+P^{1+\varepsilon}+P^{2} M^{\varepsilon-1-2^{1-k}} .
$$

For each integer $n_{0}$ satisfying $1 \leqslant n_{0} \leqslant 4 N$, it follows from Dirichlet's approximation theorem that there exist $d \in \mathbb{Z}$ and $t \in \mathbb{N}$ with

$$
(d, t)=1, \quad 1 \leqslant t \leqslant P^{k-1} M^{1-k 2^{1-k}} \text { and } \quad\left|t n_{0}^{k} \alpha-d\right| \leqslant M^{k 2^{1-k}-1} P^{1-k} .
$$

Then for $l \in \mathscr{L}$, we find from (3.13) that

$$
\begin{aligned}
|s l d-t c| & \leqslant s l M^{k 2^{1-k}-1} P^{1-k}+\frac{1}{2} t M^{k 2^{1-k}-1} P^{1-k} \\
& \leqslant \frac{1}{2}+4 P^{2-k} M^{2\left(k 2^{1-k}-1\right)}<1 .
\end{aligned}
$$

Thus we deduce that $c /(s l)=d / t$ and $s=t /(t, l)$. Therefore, on writing

$$
Z=P_{0}^{k-1} M\left|n_{0}^{k} \alpha-d / t\right|
$$

we arrive at the estimate

$$
\begin{aligned}
\sum_{l \in \mathscr{L}} T\left(n_{0}, l\right) & \ll \sum_{l \in \mathscr{L}} \frac{t^{\varepsilon} w_{k}(t /(t, l)) P n_{0}^{-1}}{1+Z l} \\
& \ll \frac{P}{n_{0}} t^{\varepsilon} \sum_{t_{0} \mid t} w_{k}\left(t / t_{0}\right) \sum_{1 \leqslant l^{\prime} \leqslant 4 N /\left(n_{0} t_{0}\right)}\left(1+Z t_{0} l^{\prime}\right)^{-1} .
\end{aligned}
$$

The innermost sum in the last expression satisfies the inequality

$$
\begin{aligned}
\sum_{1 \leqslant l^{\prime} \leqslant 4 N /\left(n_{0} t_{0}\right)}\left(1+Z t_{0} l^{\prime}\right)^{-1} & \ll \min \left\{N\left(n_{0} t_{0}\right)^{-1},\left(Z t_{0}\right)^{-1} \log P\right\} \\
& \ll N(\log P)\left(n_{0} t_{0}\right)^{-1}\left(1+Z N / n_{0}\right)^{-1} .
\end{aligned}
$$

Thus, on applying Lemma 2.2 via the upper bound $v_{k}(d) \leqslant d$ again, we obtain the estimate

$$
\sum_{l \in \mathscr{L}} T\left(n_{0}, l\right) \ll T_{1}\left(n_{0}\right),
$$

where

$$
T_{1}\left(n_{0}\right)=\frac{t^{\varepsilon} w_{k}(t) P n_{0}^{-2} N \log P}{1+P_{0}^{k}\left|n_{0}^{k} \alpha-d / t\right|} .
$$

On one hand we have the trivial estimate

$$
T_{1}\left(n_{0}\right) \ll P^{1+\varepsilon} N n_{0}^{-2} .
$$

But in view of (2.2), one has also the bound

$$
T_{1}\left(n_{0}\right) \ll \frac{P^{1+\varepsilon} N n_{0}^{-2}}{\left(t+P_{0}^{k}\left|t n_{0}^{k} \alpha-d\right|\right)^{1 / k}} .
$$


Then

$$
T_{1}\left(n_{0}\right) \ll P^{2+\varepsilon} M^{-1-2^{1-k}} n_{0}^{-1},
$$

except possibly when

$$
1 \leqslant t \leqslant M^{k 2^{1-k}} \text { and }\left|t n_{0}^{k} \alpha-d\right| \leqslant M^{k 2^{1-k}} P^{-k} .
$$

Accordingly, we define $\mathscr{N}_{0}$ to be the set of natural numbers $n_{0}$, with $1 \leqslant n_{0} \leqslant M^{2^{1-k}}$, such that the conditions (3.18) are satisfied. Then by (3.14) and (3.16) we obtain

$$
S_{3} \ll \sum_{n_{0} \in \mathcal{N}_{0}} T_{1}\left(n_{0}\right)+\sum_{n_{0}>M^{2^{1-k}}} P^{1+\varepsilon} N n_{0}^{-2}+\sum_{1 \leqslant n_{0} \leqslant 4 N} P^{2+\varepsilon} M^{-1-2^{1-k}} n_{0}^{-1},
$$

whence by (3.15),

$$
S_{1} \ll \sum_{n_{0} \in \mathscr{N}_{0}} T_{1}\left(n_{0}\right)+P^{1+\varepsilon}+P^{2+\varepsilon} M^{-1-2^{1-k}} .
$$

Suppose next that $n_{0} \in \mathscr{N}_{0}$. When $a \in \mathbb{Z}$ and $q \in \mathbb{N}$ satisfy (3.1), it follows from (3.18) that

$$
\begin{aligned}
\left|n_{0}^{k} t a-d q\right| & \leqslant n_{0}^{k} t P^{-k / 2}+q M^{k 2^{1-k}} P^{-k} \\
& \leqslant M^{k 2^{2-k}} P^{-k / 2}+M^{k 2^{1-k}} P^{-k / 2}<1 .
\end{aligned}
$$

Thus we have $d /\left(t n_{0}^{k}\right)=a / q$ and $t=q /\left(q, n_{0}^{k}\right)$, and hence by (3.17) one has

$$
\begin{aligned}
\sum_{n_{0} \in \mathcal{N}_{0}} T_{1}\left(n_{0}\right) & \ll \sum_{n_{0} \in \mathcal{N}_{0}} \frac{q^{\varepsilon} w_{k}\left(q /\left(q, n_{0}^{k}\right)\right) P n_{0}^{-2} N \log P}{1+P^{k}|\alpha-a / q|} \\
& \ll \frac{q^{\varepsilon} P N \log P}{1+P^{k}|\alpha-a / q|} \sum_{1 \leqslant n_{0} \leqslant M^{2^{1-k}}} w_{k}\left(q /\left(q, n_{0}^{k}\right)\right) n_{0}^{-2} .
\end{aligned}
$$

But on applying Lemma 2.3 in order to estimate the latter sum, we conclude that

$$
\sum_{n_{0} \in \mathcal{N}_{0}} T_{1}\left(n_{0}\right) \ll \frac{q^{\varepsilon} w_{k}(q) P N(\log P)^{2}}{1+P^{k}|\alpha-a / q|} .
$$

We now collect together (3.2), (3.3), (3.19) and (3.20), concluding that

$$
\begin{aligned}
\left|\sum_{M<m \leqslant M^{\prime}} a_{m} \sum_{\substack{P / m<n \leqslant P^{\prime} / m \\
U<n \leqslant U^{\prime}}} b_{n} e\left((m n)^{k} \alpha\right)\right|^{2} \\
\ll P^{1+\varepsilon} M+P^{2} M^{\varepsilon-2^{1-k}}+\frac{q^{\varepsilon} w_{k}(q) P^{2}(\log P)^{7}}{1+P^{k}|\alpha-a / q|},
\end{aligned}
$$

whence the conclusion of the lemma follows immediately.

Next we turn our attention to the estimation of a trilinear exponential sum. 
Lemma 3.2. Let $k$ be a natural number with $k \geqslant 4$, and let $P, P^{\prime}, M$ and $N$ be real numbers with $M \geqslant 1, N \geqslant 1,2 \leqslant P \leqslant P^{\prime} \leqslant 2 P$,

$$
M^{1-2^{2-k}} N^{2-2^{2-k}} \leqslant P^{1-2^{2-k}} \text { and } M^{2-2^{2-k}} N^{-2^{2-k}} \leqslant P^{1-2^{2-k}} .
$$

Suppose that $\left(a_{m}\right),\left(b_{n}\right)$ and $\left(c_{l}\right)$ are sequences of complex numbers satisfying

$$
\left|a_{m}\right| \leqslant 1+\log m \text { and }\left|b_{n}\right| \leqslant 1
$$

for each $m$ and $n$, and with $c_{l}=1$ for all $l$, or $c_{l}=\log l$ for all $l$. Suppose further that $\alpha$ is a real number, and that there exist $a \in \mathbb{Z}$ and $q \in \mathbb{N}$ satisfying (3.1). Then one has

$$
\begin{aligned}
\sum_{1 \leqslant m \leqslant M} a_{m} & \sum_{1 \leqslant n \leqslant N} b_{n} \sum_{P /(m n)<l \leqslant P^{\prime} /(m n)} c_{l} e\left((\operatorname{lmn})^{k} \alpha\right) \\
& \ll P^{1-2^{1-k}+\varepsilon}(M N)^{2^{1-k}}+\frac{q^{\varepsilon} w_{k}(q) P(\log P)^{4}}{1+P^{k}|\alpha-a / q|} .
\end{aligned}
$$

Proof. For each integer $m$ with $1 \leqslant m \leqslant M$, denote by $\mathscr{N}$ the set of natural numbers $n$ with $1 \leqslant n \leqslant N$ for which there exist $b \in \mathbb{Z}$ and $r \in \mathbb{N}$ with

$$
\begin{aligned}
& (b, r)=1, \quad 1 \leqslant r \leqslant \frac{1}{3}(P /(m n))^{k 2^{1-k}}, \\
& \left|r(m n)^{k} \alpha-b\right| \leqslant \frac{1}{2}(P /(m n))^{k\left(2^{1-k}-1\right)} .
\end{aligned}
$$

Then in view of Lemma 2.1, one has

$$
\sum_{1 \leqslant m \leqslant M} a_{m} \sum_{1 \leqslant n \leqslant N} b_{n} \sum_{P /(m n)<l \leqslant P^{\prime} /(m n)} c_{l} e\left((l m n)^{k} \alpha\right) \ll E_{0}+E_{1},
$$

where

and

$$
E_{0}=\sum_{1 \leqslant m \leqslant M} \sum_{1 \leqslant n \leqslant N}\left|a_{m} b_{n}\right|(P /(m n))^{1-2^{1-k}+\varepsilon}
$$

$$
E_{1}=\sum_{1 \leqslant m \leqslant M} \sum_{n \in \mathcal{N}}\left|a_{m} b_{n}\right| \frac{w_{k}(r) P(m n)^{-1} \log P}{1+(P /(m n))^{k}\left|(m n)^{k} \alpha-b / r\right|} .
$$

In view of our hypotheses concerning $\left(a_{m}\right)$ and $\left(b_{n}\right)$, one plainly has

Also, it is evident that

$$
E_{0} \ll P^{1-2^{1-k}+\varepsilon}(M N)^{2^{1-k}} .
$$

where

$$
E_{1} \ll P(\log P)^{2} E_{2},
$$

$$
E_{2}=\sum_{1 \leqslant m \leqslant M} \sum_{n \in \mathcal{N}} \frac{w_{k}(r)(m n)^{-1}}{1+(P /(m n))^{k}\left|(m n)^{k} \alpha-b / r\right|} .
$$

We now follow a similar path to that taken in the proof of the previous lemma. For each integer $m$ with $1 \leqslant m \leqslant M$, we apply Dirichlet's approximation theorem to deduce the existence of $c \in \mathbb{Z}$ and $s \in \mathbb{N}$ with

$$
\begin{gathered}
(c, s)=1, \quad 1 \leqslant s \leqslant(P /(m N))^{k\left(1-2^{1-k}\right)}, \\
\left|s m^{k} \alpha-c\right| \leqslant(P /(m N))^{k\left(2^{1-k}-1\right)} .
\end{gathered}
$$


By combining (3.22) and (3.27), we obtain

$$
\begin{aligned}
\left|r n^{k} c-s b\right| & \leqslant r n^{k}(P /(m N))^{k\left(2^{1-k}-1\right)}+\frac{1}{2} s(P /(m n))^{k\left(2^{1-k}-1\right)} \\
& \leqslant \frac{1}{2}+\frac{1}{3}(P / m)^{k\left(2^{2-k}-1\right)}(n N)^{k\left(1-2^{1-k}\right)},
\end{aligned}
$$

whence by (3.21) it follows that

$$
\left|r n^{k} c-s b\right|<1 .
$$

Thus we have

$$
\frac{b}{r n^{k}}=\frac{c}{s}, \quad r=\frac{s}{\left(s, n^{k}\right)}
$$

and so by (3.26),

$$
E_{2}=\sum_{1 \leqslant m \leqslant M} m^{-1}\left(1+(P / m)^{k}\left|m^{k} \alpha-c / s\right|\right)^{-1} \sum_{n \in \mathcal{N}} w_{k}\left(s /\left(s, n^{k}\right)\right) n^{-1} .
$$

The innermost sum may be evaluated by means of Lemma 2.3, and thus we obtain

$$
E_{2} \ll \sum_{1 \leqslant m \leqslant M} \frac{s^{\varepsilon} w_{k}(s) \log P}{m\left(1+(P / m)^{k}\left|m^{k} \alpha-c / s\right|\right)} .
$$

We next define $\mathscr{M}$ to be the set of natural numbers $m$ with $1 \leqslant m \leqslant M$ such that the integers $c$ and $s$ defined in (3.27) satisfy

$$
\begin{gathered}
1 \leqslant s \leqslant \frac{1}{3}(P /(M N))^{k 2^{1-k}}, \\
\left|s m^{k} \alpha-c\right| \leqslant \frac{1}{3}(P /(M N))^{k 2^{1-k}}(P / m)^{-k} .
\end{gathered}
$$

In view of (2.2) and (3.28), we find that

$$
E_{2} \ll \sum_{m \in \mathscr{M}} \frac{s^{\varepsilon} w_{k}(s) \log P}{m\left(1+(P / m)^{k}\left|m^{k} \alpha-c / s\right|\right)}+P^{\varepsilon-2^{1-k}}(M N)^{2^{1-k}} .
$$

When $a$ and $q$ satisfy (3.1) and $m \in \mathscr{M}$, it follows from (3.29) that

$$
\begin{aligned}
\left|s m^{k} a-q c\right| & \leqslant s m^{k} P^{-k / 2}+\frac{1}{3} q(P /(M N))^{k 2^{1-k}}(P / m)^{-k} \\
& \leqslant \frac{2}{3} P^{\frac{1}{2} k\left(2^{2-k}-1\right)} M^{k\left(1-2^{1-k}\right)} N^{-k 2^{1-k}}
\end{aligned}
$$

whence by the second condition of (3.21) we obtain

$$
\left|s m^{k} a-q c\right|<1 .
$$

Consequently,

$$
\frac{c}{s m^{k}}=\frac{a}{q} \quad \text { and } \quad s=\frac{q}{\left(q, m^{k}\right)},
$$

and thus by Lemma 2.3 , we deduce that

$$
\begin{aligned}
\sum_{m \in \mathscr{M}} \frac{s^{\varepsilon} w_{k}(s) \log P}{m\left(1+(P / m)^{k}\left|m^{k} \alpha-c / s\right|\right)} & \leqslant \frac{q^{\varepsilon} \log P}{1+P^{k}|\alpha-a / q|} \sum_{1 \leqslant m \leqslant M} \frac{w_{k}\left(q /\left(q, m^{k}\right)\right)}{m} \\
& \ll \frac{q^{\varepsilon} w_{k}(q)(\log P)^{2}}{1+P^{k}|\alpha-a / q|}
\end{aligned}
$$


The proof of the lemma is completed by collecting together (3.23), (3.24), (3.25), (3.30) and (3.31).

Finally, we provide an estimate for an exponential sum over prime numbers.

Lemma 3.3. Let $k$ be a natural number with $k \geqslant 4$, and let $P$ be a real number with $P \geqslant 2$. Suppose that $\alpha$ is a real number, and that there exist $a \in \mathbb{Z}$ and $q \in \mathbb{N}$ satisfying (3.1). Then one has

$$
\sum_{P<p \leqslant 2 P} e\left(p^{k} \alpha\right) \ll P^{1-2^{-k-1}+\varepsilon}+\frac{q^{\varepsilon} w_{k}(q)^{1 / 2} P(\log P)^{4}}{\left(1+P^{k}|\alpha-a / q|\right)^{1 / 2}} .
$$

Proof. We begin by observing that it is sufficient to estimate a weighted exponential sum. For on writing

$$
S(t)=\sum_{P<p \leqslant t}(\log p) e\left(p^{k} \alpha\right)
$$

one finds by partial summation that

$$
\sum_{P<p \leqslant 2 P} e\left(p^{k} \alpha\right)=\frac{S(2 P)}{\log (2 P)}+\int_{P}^{2 P} \frac{S(t)}{t(\log t)^{2}} d t .
$$

In order to establish the lemma, therefore, it suffices to show that for each real number $P^{\prime}$ with $P \leqslant P^{\prime} \leqslant 2 P$, one has

where

$$
S\left(P^{\prime}\right) \ll P^{1-2^{-k-1}+\varepsilon}+\Phi,
$$

$$
\Phi=\frac{q^{\varepsilon} w_{k}(q)^{1 / 2} P(\log P)^{5}}{\left(1+P^{k}|\alpha-a / q|\right)^{1 / 2}} .
$$

Let $\mu(n)$ denote the Möbius function. Also, let $\Lambda(n)$ denote the von Mangoldt function, defined to be $\log p$ whenever $n$ is the prime power $p^{h}$, and zero otherwise. Then plainly, whenever $P \leqslant P^{\prime} \leqslant 2 P$, one has

$$
S\left(P^{\prime}\right)=\sum_{P<n \leqslant P^{\prime}} \Lambda(n) e\left(n^{k} \alpha\right)+O\left(P^{1 / 2}\right) .
$$

We next appeal to Vaughan's identity (see, for example, Vaughan [16]), with $U=P^{1 / 4}$ and $V=P^{1 / 2}$, to show that whenever $P \leqslant n \leqslant 2 P$, one has

$$
\Lambda(n)=\sum_{\substack{d m=n \\ 1 \leqslant d \leqslant V}} \mu(d) \log m-\sum_{\substack{d l m=n \\ 1 \leqslant d \leqslant V \\ 1 \leqslant m \leqslant U}} \mu(d) \Lambda(m)-\sum_{\substack{d l m=n \\ 1 \leqslant d \leqslant V \\ m>U \\ d l>V}} \mu(d) \Lambda(m) .
$$

Thus we deduce that

$$
S\left(P^{\prime}\right)=S_{1}-S_{2}-S_{3}+O\left(P^{1 / 2}\right)
$$


where

$$
\begin{aligned}
S_{1} & =\sum_{1 \leqslant d \leqslant V} \mu(d) \sum_{P / d<m \leqslant P^{\prime} / d}(\log m) e\left((d m)^{k} \alpha\right), \\
S_{2} & =\sum_{1 \leqslant v \leqslant U V} \lambda_{0}(v) \sum_{\substack{P / v<l \leqslant P^{\prime} / v \\
V}} e\left((v l)^{k} \alpha\right), \\
S_{3} & =\sum_{V<u \leqslant P^{\prime} / U} \lambda_{1}(u) \sum_{\substack{P / u<m \leqslant P^{\prime} / u \\
m>U}} \Lambda(m) e\left((u m)^{k} \alpha\right),
\end{aligned}
$$

and here we write

$$
\lambda_{0}(v)=\sum_{\substack{d m=v \\ 1 \leq d \leq V \\ 1 \leqslant m \leqslant U}} \mu(d) \Lambda(m) \quad \text { and } \quad \lambda_{1}(u)=\sum_{\substack{d \mid u \\ 1 \leqslant d \leqslant V}} \mu(d) .
$$

Notice in particular that

$$
\left|\lambda_{1}(u)\right| \leqslant \tau(u) \quad \text { and } \quad\left|\lambda_{0}(v)\right| \leqslant \sum_{m \mid v} \Lambda(m)=\log v .
$$

The exponential sum $S_{3}$ may be estimated simply by means of Lemma 3.1, owing to the conditions (3.38). Thus, by dividing the summation over $u$ in (3.37) into dyadic intervals, we deduce that

$$
S_{3} \ll(\log P) \max _{V \leqslant M \leqslant P^{\prime} / U}\left(P M^{\varepsilon-2^{-k}}+(P M)^{1 / 2+\varepsilon}+(\log P)^{-1} \Phi\right),
$$

where $\Phi$ is defined in (3.33). In view of our choices for $U$ and $V$, therefore, one finds that

$$
S_{3} \ll P^{1-2^{-k-1}+\varepsilon}+\Phi
$$

Next we estimate $S_{2}$. Write

$$
S_{4}(Y, Z)=\sum_{Y<v \leqslant Z} \lambda_{0}(v) \sum_{P / v<l \leqslant P^{\prime} / v} e\left((v l)^{k} \alpha\right)
$$

Then by (3.36) we plainly have

$$
S_{2}=S_{4}(0, U)+S_{4}(U, V)+S_{4}(V, U V) .
$$

In view of the conditions (3.38), we may again divide the summation over $v$ into dyadic intervals to deduce from Lemma 3.1 that

$$
S_{4}(V, U V) \ll P^{1-2^{-k-1}+\varepsilon}+\Phi .
$$

Moreover, one may rearrange the summation in $S_{4}(U, V)$ to obtain

$$
S_{4}(U, V)=\sum_{P / V<l \leqslant P^{\prime} / U} \sum_{\substack{P / l<v \leqslant P^{\prime} / l \\ U<v \leqslant V}} \lambda_{0}(v) e\left((l v)^{k} \alpha\right),
$$

whence a dyadic dissection of the summation over $l$ now yields, again by Lemma 3.1,

$$
S_{4}(U, V) \ll P^{1-2^{-k-1}+\varepsilon}+\Phi .
$$

In order to estimate $S_{4}(0, U)$, we note that $U^{2-2^{2-k}} \leqslant P^{1-2^{2-k}}$, so that an 
application of Lemma 3.2 with $N=1$ and $b_{1}=1$ yields

$$
S_{4}(0, U) \ll P^{1-2^{1-k}+\varepsilon} U^{2^{1-k}}+\Phi \ll P^{1-2^{-k}+\varepsilon}+\Phi .
$$

On collecting together (3.40)-(3.43), we obtain

$$
S_{2} \ll P^{1-2^{-k-1}+\varepsilon}+\Phi .
$$

Finally, in order to estimate $S_{1}$, we write

$$
S_{5}(Y, Z)=\sum_{Y<d \leqslant Z} \mu(d) \sum_{P / d<m \leqslant P^{\prime} / d}(\log m) e\left((d m)^{k} \alpha\right),
$$

and then observe that by (3.35) we have

$$
S_{1}=S_{5}(0, U)+S_{5}(U, V) .
$$

But again rearranging the summation in $S_{5}(U, V)$, we obtain

$$
S_{5}(U, V)=\sum_{P / V<m \leqslant P^{\prime} / U}(\log m) \sum_{\substack{P / m<d \leqslant P^{\prime} / m \\ U<d \leqslant V}} \mu(d) e\left((m d)^{k} \alpha\right),
$$

whence a dyadic dissection of the summation over $m$ now yields, by Lemma 3.1,

$$
S_{5}(U, V) \ll P^{1-2^{-k-1}+\varepsilon}+\Phi .
$$

Also, noting again that $U^{2-2^{2-k}} \leqslant P^{1-2^{2-k}}$, an application of Lemma 3.2 with $N=1$ and $b_{1}=1$ reveals that

$$
S_{5}(0, U) \ll P^{1-2^{1-k}+\varepsilon} U^{2^{1-k}}+\Phi \ll P^{1-2^{-k}+\varepsilon}+\Phi .
$$

Then from (3.45)-(3.47) we have

$$
S_{1} \ll P^{1-2^{-k-1}+\varepsilon}+\Phi
$$

The proof of the lemma is completed on combining (3.34), (3.39), (3.44) and (3.48), and recalling our discussion surrounding (3.32).

\section{Mean value estimates for exponential sums, $I$}

In advance of our application of the Hardy-Littlewood method for sums of powers of prime numbers, we require estimates for the mean values of suitable exponential sums. In this section we concentrate on providing such bounds for exponential sums over fourth powers, though certain of these estimates remain relevant to our later investigations concerning fifth powers.

We begin by recalling a result from Vaughan [17].

Lemma 4.1. Suppose that

$$
\begin{gathered}
k \geqslant 4, \quad s=\left[\frac{1}{2}(k+3)\right], \\
\mu_{1}=1, \quad 1 \geqslant \mu_{2} \geqslant 1-1 / k, \quad \mu_{2} \geqslant \mu_{j}>\frac{1}{2} \quad(2 \leqslant j \leqslant s), \\
P_{j}=P^{\mu_{j}}, \quad \nu=k \mu_{2}-k+1, \quad C=C(k, \varepsilon) \geqslant 2^{k} .
\end{gathered}
$$


Let $R(m)$ be a non-negative arithmetical function, and define

Then

$$
\begin{aligned}
f_{0}(\alpha) & =\sum_{0 \leqslant m \leqslant C P_{2}^{k}} R(m) e(m \alpha), \\
f_{j}(\alpha) & =\sum_{P_{j}<x \leqslant 2 P_{j}} e\left(\alpha x^{k}\right) \quad(1 \leqslant j \leqslant s), \\
F_{j}(\alpha) & =f_{j}(\alpha) f_{j+1}(\alpha) \ldots f_{s}(\alpha) f_{0}(\alpha) \quad(j=1,2) .
\end{aligned}
$$

$$
\int_{0}^{1}\left|F_{1}(\alpha)\right|^{2} d \alpha \ll\left(P+P^{1+\nu-2^{2-k}+\varepsilon}\right) \int_{0}^{1}\left|F_{2}(\alpha)\right|^{2} d \alpha+F_{1}(0)^{2} P^{-k}(\log P)^{2} .
$$

Proof. On consideration of the underlying diophantine equations, the conclusion of the lemma is essentially immediate from Theorem 3 of Vaughan [17], save that therein, the factor $(\log P)^{2}$ appearing above is replaced by $P^{\varepsilon}$. In order to replace Vaughan's conclusion with the slightly sharper one above, a careful examination of the proof of [17, Theorem 3] reveals that one must modify Vaughan's treatment of the underlying major arc contribution. Adopting the notation of [17] for the sake of conciseness, one observes that the argument culminating at the top of p. 450 of [17] yields

$$
\begin{aligned}
G(\alpha) & =q^{\varepsilon-1 /(k-2)} \sum_{1 \leqslant h \leqslant H}\left(\frac{(q, h) P}{1+|\beta| h P^{k-1}}+q(q, h)^{1 /(k-1)}\right) \\
& \ll q^{\varepsilon-1 /(k-2)} P^{1+\nu}(\log P)\left(1+|\beta| P^{\nu+k-1}\right)^{-1}+P^{\nu} q^{(k-2) /(k-1)+\varepsilon},
\end{aligned}
$$

saving a factor of $P^{\varepsilon}$ over the corresponding estimate recorded in Lemma 2 of [17]. On substituting the estimate (4.1) within the argument presented on pages 450-452 of [17], one finds that the mean value $K$ defined in (2.21) of [17] satisfies

$$
K \ll(\log P)^{2} P^{-k}\left(\sum_{m} R_{1}(m)\right)^{2},
$$

the additional logarithmic factor arising in the evaluation of the mean value

$$
\int_{\mathfrak{N}(q, a)} G_{1}(\alpha)|F(\alpha)|^{2} d \alpha
$$

at the top of p. 452 of [17]. The estimate (4.2) suffices to establish the lemma in its stated form.

We prepare for later use a straightforward estimate of considerable utility.

LeMma 4.2. Let $k$ be a natural number with $k \geqslant 4$, and write $t=\left[\frac{1}{2} k\right]$. Let $\mu_{j}$ $(1 \leqslant j \leqslant t)$ be real numbers with $\mu_{1}=1$ and $\frac{1}{2}<\mu_{j} \leqslant 1(2 \leqslant j \leqslant t)$. Also, let $P_{j}$ and $f_{j}(\alpha)$ be as defined in the statement of Lemma 4.1, and let $g(\alpha)$ be a complex-valued function, periodic with period 1 , and having the property $|g(\alpha)| \leqslant g(0)$ for $\alpha \in \mathbb{R}$. Finally, write

$$
F(\alpha)=f_{1}(\alpha) f_{2}(\alpha) \ldots f_{t}(\alpha) g(\alpha) .
$$


Then

$$
\int_{0}^{1}\left|f_{1}(\alpha) F(\alpha)\right|^{2} d \alpha \ll P^{2-2^{2-k}+\varepsilon} \int_{0}^{1}|F(\alpha)|^{2} d \alpha+F(0)^{2} P^{2-k} \log P .
$$

Proof. We apply the Hardy-Littlewood method. Write $Q=P^{k 2^{1-k}}$, and recalling the notation defined in (2.15) and (2.16), write

$$
\mathfrak{M}(q, a)=\mathfrak{M}\left(q, a ; Q ; P^{k}\right), \quad \mathfrak{M}=\mathfrak{M}\left(Q ; P^{k}\right) \quad \text { and } \quad \mathfrak{m}=\mathfrak{m}\left(Q ; P^{k}\right) .
$$

By Lemma 2.1, we have

$$
\sup _{\alpha \in \mathfrak{m}}\left|f_{1}(\alpha)\right| \ll P^{1-2^{1-k}+\varepsilon},
$$

and when $\alpha \in \mathfrak{M}(q, a) \subseteq \mathfrak{M}$, we have

Consequently,

$$
f_{1}(\alpha) \ll w_{k}(q) P\left(1+P^{k}|\alpha-a / q|\right)^{-1} .
$$

$$
\int_{0}^{1}\left|f_{1}(\alpha) F(\alpha)\right|^{2} d \alpha \ll P^{2-2^{2-k}+\varepsilon} \int_{0}^{1}|F(\alpha)|^{2} d \alpha+S,
$$

where

$$
S=g(0)^{2} \sum_{1 \leqslant q \leqslant Q} \sum_{\substack{a=1 \\(a, q)=1}}^{q} \int_{\mathfrak{M}(q, a)} \frac{w_{k}(q)^{4} P^{4}}{\left(1+P^{k}|\alpha-a / q|\right)^{4}}\left|f_{2}(\alpha) \ldots f_{t}(\alpha)\right|^{2} d \alpha .
$$

But since $Q \leqslant P^{1 / 2}$ and $\mu_{j}>\frac{1}{2}(1 \leqslant j \leqslant t)$, we may apply Lemmata 6.1 and 6.2 of [19] in combination with (2.2) and (2.3) to conclude that for $\alpha \in \mathfrak{M}(q, a) \subseteq \mathfrak{M}$, one has

$$
f_{j}(\alpha) \ll w_{k}(q) P_{j}+q^{1 / 2+\varepsilon} \ll w_{k}(q) P_{j} .
$$

Thus we deduce that

$$
\begin{aligned}
S & \ll P^{4} P_{2}^{2} \ldots P_{t}^{2} g(0)^{2} \sum_{1 \leqslant q \leqslant Q} q w_{k}(q)^{2 t+2} \int_{0}^{\infty}\left(1+P^{k} \beta\right)^{-4} d \beta \\
& \ll F(0)^{2} P^{2-k} \sum_{1 \leqslant q \leqslant Q} q w_{k}(q)^{k+1} .
\end{aligned}
$$

The proof of the lemma is completed by making use of Lemma 2.4 to estimate the sum on the right-hand side of (4.4), and then substituting the conclusion into (4.3).

For the remainder of this section, and the next, we restrict attention to the case $k=4$, and fix notation as follows. We put

$$
\begin{gathered}
\lambda_{1}=1, \quad \lambda_{2}=\frac{13}{16}, \quad \lambda_{3}=\left(\frac{13}{16}\right)^{2}, \quad \lambda_{4}=\left(\frac{13}{16}\right)^{2} \frac{91}{111}, \quad \lambda_{5}=\left(\frac{13}{16}\right)^{2} \frac{78}{111}, \\
P_{j}=P^{\lambda_{j}}, \quad f_{j}(\alpha)=\sum_{P_{j}<x \leqslant 2 P_{j}} e\left(\alpha x^{4}\right) \quad(1 \leqslant j \leqslant 5), \\
\mathscr{F}_{j}(\alpha)=f_{5}(\alpha)^{2} \prod_{i=j}^{4} f_{i}(\alpha) \quad(1 \leqslant j \leqslant 4) .
\end{gathered}
$$


We remark that the exponents $\lambda_{j}$ recorded in (4.5) have been chosen in accordance with Theorem 3 of Thanigasalam [14], which we record as the following lemma.

Lemma 4.3. For $1 \leqslant j \leqslant 4$, one has

$$
\int_{0}^{1}\left|\mathscr{F}_{j}(\alpha)\right|^{2} d \alpha \ll P^{\varepsilon} \mathscr{F}_{j}(0) .
$$

We complete this section by establishing some auxiliary mean value estimates of use in certain pruning procedures.

Lemma 4.4. When $j$ is an integer with $1 \leqslant j \leqslant 5$, write

$$
I_{j}=\int_{0}^{1}\left|f_{j}(\alpha) \mathscr{F}_{1}(\alpha)\right|^{2} d \alpha .
$$

Then for $1 \leqslant j \leqslant 5$, one has

$$
I_{j} \ll \mathscr{F}_{1}(0)^{2} P_{j}^{2} P^{-4}(\log P)^{2} .
$$

Proof. The lemma follows simply by applying Lemmata 4.1-4.3. Before proceeding further, it is useful to make some preliminary evaluations of pertinent parameters. We define the numbers $\nu_{j}$, for $2 \leqslant j \leqslant 5$, by $P_{j-1}^{\nu_{j}}=P_{j}^{4} P_{j-1}^{-3}$. Thus

Write

$$
\nu_{2}=\nu_{3}=\frac{1}{4}, \quad \nu_{4}=\frac{31}{111} \quad \text { and } \quad \nu_{5}=\frac{3}{7} .
$$

$$
X_{j}=P_{j}^{-1 / 4}(1 \leqslant j \leqslant 3), \quad X_{4}=P_{3}^{\nu_{4}-1 / 4} P_{4}^{-1 / 4}, \quad X_{5}=P_{3}^{\nu_{4}-1 / 4} P_{4}^{\nu_{5}-1 / 4} P_{5}^{-1 / 2} \text {. }
$$

Then since $\nu_{4}>\frac{1}{4}$ and $P_{5}^{1 / 2}=P_{4}^{3 / 7}=P_{4}^{\nu_{5}}$, we see at once that

$$
X_{1}<X_{2}<X_{3}<X_{4}=X_{5} \text {. }
$$

Moreover, in view of (4.5)-(4.7), a modest calculation reveals that

$$
\mathscr{F}_{1}(0) \asymp P^{56003 / 14208}>P^{4-0.05835},
$$

and also $P^{4} \mathscr{F}_{1}(0)^{-1} X_{4} \leqslant P^{-0.0576}$, whence

$$
P^{4+\varepsilon} \mathscr{F}_{1}(0)^{-1} X_{j}<1 \quad(1 \leqslant j \leqslant 5) .
$$

Next put

$$
Y_{2}=P^{3} P_{2}^{-4}, \quad Y_{3}=P^{3} P_{2}^{-1} P_{3}^{-4},
$$

and

$$
Y_{4}=P^{3} P_{2}^{-1} P_{3}^{\nu_{4}-5 / 4} P_{4}^{-4}
$$

We observe that by the definition of the $\nu_{j}$, one has $Y_{j}=\left(P_{1} \ldots P_{j-1}\right)^{-1 / 4}$ for $2 \leqslant j \leqslant 4$, whence

$$
Y_{j} P^{\varepsilon}<1 \quad(2 \leqslant j \leqslant 4) .
$$

We now initiate our main argument, establishing the lemma for each $j$ in turn. Applying Lemma 4.2 to $I_{1}$, and then appealing to Lemma 4.3, we obtain

$$
\begin{aligned}
I_{1} & \ll P^{2-1 / 4+\varepsilon} \mathscr{F}_{1}(0)+\mathscr{F}_{1}(0)^{2} P^{-2} \log P \\
& \ll \mathscr{F}_{1}(0)^{2} P^{-2}(\log P)\left(P^{4+\varepsilon} \mathscr{F}_{1}(0)^{-1} X_{1}+1\right) .
\end{aligned}
$$

Then in view of (4.9), the conclusion of the lemma holds when $j=1$. 
Next applying Lemma 4.1 to estimate $I_{2}$, and then employing Lemmata 4.2 and 4.3 in order, we obtain

$$
\begin{aligned}
I_{2} & =\int_{0}^{1}\left|f_{1}(\alpha) f_{2}(\alpha) \mathscr{F}_{2}(\alpha)\right|^{2} d \alpha \\
& \ll P^{1+\varepsilon}\left(P_{2}^{2-1 / 4+\varepsilon} \mathscr{F}_{2}(0)+\mathscr{F}_{2}(0)^{2} P_{2}^{-2} \log P\right)+\mathscr{F}_{1}(0)^{2} P_{2}^{2} P^{-4}(\log P)^{2} \\
& \ll \mathscr{F}_{1}(0)^{2} P_{2}^{2} P^{-4}\left(P^{4+\varepsilon} \mathscr{F}_{1}(0)^{-1} X_{2}+Y_{2} P^{\varepsilon}+(\log P)^{2}\right) .
\end{aligned}
$$

Then by (4.9) and (4.10), the conclusion of the lemma holds also when $j=2$.

Similarly, we utilise Lemma 4.1 twice, and then apply Lemmata 4.2 and 4.3 to reveal that

$$
\begin{aligned}
I_{3} & =\int_{0}^{1}\left|f_{1}(\alpha) f_{2}(\alpha) f_{3}(\alpha) \mathscr{F}_{3}(\alpha)\right|^{2} d \alpha \\
& \ll P P_{2} P_{3}^{2-1 / 4+\varepsilon} \mathscr{F}_{3}(0)+\mathscr{F}_{1}(0)^{2} P_{3}^{2} P^{-4}\left(\left(Y_{3}+Y_{2}\right) P^{\varepsilon}+(\log P)^{2}\right) \\
& \ll \mathscr{F}_{1}(0)^{2} P_{3}^{2} P^{-4}\left(P^{4+\varepsilon} \mathscr{F}_{1}(0)^{-1} X_{3}+\left(Y_{3}+Y_{2}\right) P^{\varepsilon}+(\log P)^{2}\right) .
\end{aligned}
$$

The desired bound for $j=3$ therefore follows again from (4.9) and (4.10).

Repeating the application of Lemma 4.1 thrice, and then applying Lemmata 4.2 and 4.3 again, we now obtain

$$
\begin{aligned}
I_{4}= & \int_{0}^{1}\left|f_{1}(\alpha) f_{2}(\alpha) f_{3}(\alpha) f_{4}(\alpha) \mathscr{F}_{4}(\alpha)\right|^{2} d \alpha \\
\ll & P P_{2} P_{3}^{1+\nu_{4}-1 / 4} P_{4}^{2-1 / 4+\varepsilon} \mathscr{F}_{4}(0) \\
& +\mathscr{F}_{1}(0)^{2} P_{4}^{2} P^{-4}\left(\left(Y_{4}+Y_{3}+Y_{2}\right) P^{\varepsilon}+(\log P)^{2}\right) \\
& \ll \mathscr{F}_{1}(0)^{2} P_{4}^{2} P^{-4}\left(P^{4+\varepsilon} \mathscr{F}_{1}(0)^{-1} X_{4}+\left(Y_{4}+Y_{3}+Y_{2}\right) P^{\varepsilon}+(\log P)^{2}\right) .
\end{aligned}
$$

The desired bound for $j=4$ consequently follows again from (4.9) and (4.10).

Finally we turn to the estimation of $I_{5}$. Applying now Lemma 4.1 four times, we have

$$
\begin{aligned}
I_{5}= & \int_{0}^{1}\left|f_{1}(\alpha) f_{2}(\alpha) f_{3}(\alpha) f_{4}(\alpha) f_{5}(\alpha)^{3}\right|^{2} d \alpha \\
\ll & P P_{2} P_{3}^{1+\nu_{4}-1 / 4} P_{4}^{1+\nu_{5}-1 / 4+\varepsilon} \int_{0}^{1}\left|f_{5}(\alpha)\right|^{6} d \alpha \\
& +\mathscr{F}_{1}(0)^{2} P_{5}^{2} P^{-4}\left(\left(Y_{4}+Y_{3}+Y_{2}\right) P^{\varepsilon}+(\log P)^{2}\right) .
\end{aligned}
$$

But by Hua's inequality (see Lemma 2.5 of [19]) and Schwarz's inequality,

$$
\int_{0}^{1}\left|f_{5}(\alpha)\right|^{6} d \alpha \ll\left(\int_{0}^{1}\left|f_{5}(\alpha)\right|^{4} d \alpha\right)^{1 / 2}\left(\int_{0}^{1}\left|f_{5}(\alpha)\right|^{8} d \alpha\right)^{1 / 2} \ll P_{5}^{7 / 2+\varepsilon} .
$$

Thus, by (4.9)-(4.11) we deduce that

$$
\begin{aligned}
I_{5} & \ll \mathscr{F}_{1}(0)^{2} P_{5}^{2} P^{-4}\left(P^{4+\varepsilon} \mathscr{F}_{1}(0)^{-1} X_{5}+\left(Y_{4}+Y_{3}+Y_{2}\right) P^{\varepsilon}+(\log P)^{2}\right) \\
& \ll \mathscr{F}_{1}(0)^{2} P_{5}^{2} P^{-4}(\log P)^{2},
\end{aligned}
$$

and so the desired bound follows for $j=5$.

This completes the proof of the lemma. 


\section{The Waring-Goldbach problem for fourth powers}

We now complete the proof of Theorem 1, retaining the notation introduced in the previous section. Let $s$ be a natural number with $s \geqslant 14$, and let $n$ be a sufficiently large integer with $n \equiv s(\bmod 240)$. Since $n_{1}=n-(s-14) \cdot 7^{4}$ satisfies $n_{1} \equiv 14(\bmod 240)$, we find that $n$ is represented as the sum of $s$ fourth powers of prime numbers whenever $n_{1}$ is the sum of 14 fourth powers of prime numbers. Thus, in order to establish that $H(4) \leqslant 14$, it suffices to show that all sufficiently large natural numbers $m$, with $m \equiv 14(\bmod 240)$, are the sum of 14 fourth powers of prime numbers.

Consider then a sufficiently large natural number $n$ with $n \equiv 14(\bmod 240)$, and write

$$
P=\frac{1}{2} n^{1 / 4} \text {. }
$$

Let $R(n)$ denote the number of representations of $n$ in the form

$$
n=p_{1}^{4}+p_{2}^{4}+\ldots+p_{14}^{4},
$$

with

$$
\begin{gathered}
P<p_{j} \leqslant 2 P(1 \leqslant j \leqslant 4), \quad P_{i}<p_{2 i+1}, p_{2 i+2} \leqslant 2 P_{i} \quad(2 \leqslant i \leqslant 4), \\
P_{5}<p_{l} \leqslant 2 P_{5} \quad(11 \leqslant l \leqslant 14) .
\end{gathered}
$$

We aim to apply the Hardy-Littlewood method to establish that $R(n)>0$, whence Theorem 1 follows according to the above discussion. To this end we introduce the exponential sums

$$
g_{j}(\alpha)=\sum_{P_{j}<p \leqslant 2 P_{j}} e\left(p^{4} \alpha\right) \quad(1 \leqslant j \leqslant 5)
$$

and write

$$
\mathscr{G}_{1}(\alpha)=g_{1}(\alpha) g_{2}(\alpha) g_{3}(\alpha) g_{4}(\alpha) g_{5}(\alpha)^{2} .
$$

When $\mathfrak{B} \subseteq[0,1)$, define

$$
R(n ; \mathfrak{B})=\int_{\mathfrak{B}} g_{1}(\alpha)^{2} \mathscr{G}_{1}(\alpha)^{2} e(-n \alpha) d \alpha
$$

Then by orthogonality, we have $R(n)=R(n ;[0,1))$.

Next we define the Hardy-Littlewood dissections fundamental to our application of the circle method. We take $L=(\log P)^{2000}$, and define $\mathfrak{N}$ to be the union of the intervals

$$
\mathfrak{N}(q, a)=\left\{\alpha \in[0,1):|\alpha-a / q| \leqslant L P^{-4}\right\}
$$

with $0 \leqslant a \leqslant q \leqslant L$ and $(a, q)=1$. Notice that the $\operatorname{arcs} \mathfrak{N}(q, a)$ comprising $\mathfrak{N}$ are pairwise disjoint. We write also $\mathfrak{n}=[0,1) \backslash \mathfrak{N}$, and note that

$$
R(n)=R(n ; \mathfrak{N})+R(n ; \mathfrak{n}) .
$$

We analyse the minor arcs $n$ by means of a further dissection, and in this context we recall the notation defined in (2.15) and (2.16), and write

$$
\mathfrak{M}(q, a)=\mathfrak{M}\left(q, a ; P^{1 / 4} ; P^{4}\right), \quad \mathfrak{M}=\mathfrak{M}\left(P^{1 / 4} ; P^{4}\right), \quad \mathfrak{m}=\mathfrak{m}\left(P^{1 / 4} ; P^{4}\right) .
$$

We remark that $\mathfrak{m} \subseteq \mathfrak{n}$. 
We define the function $\Psi(\alpha)$ for $\alpha \in \mathfrak{M}$ by taking

$$
\Psi(\alpha)=q^{\delta} w_{4}(q)\left(1+P^{4}|\alpha-a / q|\right)^{-1},
$$

for $\alpha \in \mathfrak{M}(q, a) \subseteq \mathfrak{M}$, where we write $\delta=10^{-2}$. In particular, it follows from (2.2) that when $\alpha \in \mathfrak{M}(q, a) \subseteq \mathfrak{M}$ and $\alpha \in \mathfrak{n}$, then one has

$$
\Psi(\alpha) \ll\left(q+P^{4}|q \alpha-a|\right)^{\delta-1 / 4} \ll L^{-1 / 5} .
$$

Consequently, by Lemma 3.3, when $\alpha \in \mathfrak{M} \cap \mathfrak{n}$ one has

$$
\begin{aligned}
g_{1}(\alpha) & \ll P^{1-1 / 32+\varepsilon}+P(\log P)^{4} \Psi(\alpha)^{1 / 2} \\
& \ll P^{1-1 / 32+\varepsilon}+P(\log P)^{4} L^{-1 / 20} \Psi(\alpha)^{1 / 4} .
\end{aligned}
$$

Meanwhile, when $\alpha \in \mathfrak{m}$, we may apply Dirichlet's approximation theorem to deduce the existence of $a \in \mathbb{Z}$ and $q \in \mathbb{N}$ satisfying the conditions (3.1), and necessarily one has either

$$
q>P^{1 / 4} \text { or }|q \alpha-a|>P^{-15 / 4} .
$$

In this situation it therefore follows from (2.2) that

$$
\frac{w_{4}(q)^{1 / 2} P}{\left(1+P^{4}|\alpha-a / q|\right)^{1 / 2}} \ll \frac{P}{\left(q+P^{4}|q \alpha-a|\right)^{1 / 8}} \ll P^{1-1 / 32},
$$

whence by Lemma 3.3,

$$
\sup _{\alpha \in \mathfrak{m}}\left|g_{1}(\alpha)\right| \ll P^{1-1 / 32+\varepsilon} .
$$

On substituting (5.3) and (5.4) into (5.1), we deduce that

$$
|R(n ; \mathfrak{n})| \leqslant \int_{\mathfrak{n}}\left|g_{1}(\alpha) \mathscr{G}_{1}(\alpha)\right|^{2} d \alpha \ll K_{1}+K_{2},
$$

where

and

$$
K_{1}=P^{2-1 / 16+\varepsilon} \int_{0}^{1}\left|\mathscr{G}_{1}(\alpha)\right|^{2} d \alpha
$$

$$
K_{2}=P^{2}(\log P)^{-150} \int_{\mathfrak{M} \cap \mathfrak{n}} \Psi(\alpha)^{1 / 2}\left|\mathscr{G}_{1}(\alpha)\right|^{2} d \alpha
$$

But on considering the underlying diophantine equations, we see from Lemma 4.3 that

$$
\int_{0}^{1}\left|\mathscr{G}_{1}(\alpha)\right|^{2} d \alpha \leqslant \int_{0}^{1}\left|\mathscr{F}_{1}(\alpha)\right|^{2} d \alpha \ll P^{\varepsilon} \mathscr{F}_{1}(0) .
$$

A comparison between the definitions of $\mathscr{F}_{1}(\alpha)$ and $\mathscr{G}_{1}(\alpha)$ reveals that

$$
\mathscr{G}_{1}(0) \asymp \mathscr{F}_{1}(0)(\log P)^{-6},
$$

and thus we deduce from (4.8) and (5.6) that

$$
K_{1} \ll \mathscr{G}_{1}(0)^{2}\left(\mathscr{F}_{1}(0)^{-1} P^{2-1 / 16+\varepsilon}\right) \ll \mathscr{G}_{1}(0)^{2} P^{-2.004} .
$$

We turn our attention next to the estimation of $K_{2}$. By applying Hölder's 
inequality within (5.7), we obtain

$$
\int_{\mathfrak{M} \cap \mathfrak{n}} \Psi(\alpha)^{1 / 2}\left|\mathscr{G}_{1}(\alpha)\right|^{2} d \alpha \ll\left(\int_{\mathfrak{M}} \Psi(\alpha)^{3}\left|g_{5}(\alpha)\right|^{2} d \alpha\right)^{1 / 6} \prod_{j=1}^{5} J_{j}^{1 / 6},
$$

where

$$
J_{j}=\int_{0}^{1}\left|g_{j}(\alpha) \mathscr{G}_{1}(\alpha)\right|^{2} d \alpha \quad(1 \leqslant j \leqslant 5) .
$$

But on recalling the definition of $I_{j}$ from the statement of Lemma 4.4, and considering the underlying diophantine equations, it follows from the latter lemma that

$$
J_{j} \leqslant I_{j} \ll \mathscr{F}_{1}(0)^{2} P_{j}^{2} P^{-4}(\log P)^{2} \quad(1 \leqslant j \leqslant 5) .
$$

Moreover, since $P^{1 / 4}<P_{5}$, we find from Lemma 2.5 that

$$
\int_{\mathfrak{M}} \Psi(\alpha)^{3}\left|g_{5}(\alpha)\right|^{2} d \alpha \leqslant \int_{\mathfrak{M}\left(P_{5} ; P^{4}\right)} \Psi(\alpha)^{3}\left|g_{5}(\alpha)\right|^{2} d \alpha \ll P_{5}^{2} P^{-4} .
$$

Thus we deduce from (5.10) that

$$
\begin{aligned}
\int_{\mathfrak{M} \cap \mathfrak{n}} \Psi(\alpha)^{1 / 2}\left|\mathscr{G}_{1}(\alpha)\right|^{2} d \alpha & \ll\left(P_{5}^{2} P^{-4}\right)^{1 / 6} \prod_{j=1}^{5}\left(\mathscr{F}_{1}(0)^{2} P_{j}^{2} P^{-4}(\log P)^{2}\right)^{1 / 6} \\
& \ll \mathscr{F}_{1}(0)^{2} P^{-4}(\log P)^{2} .
\end{aligned}
$$

Consequently, on recalling (5.8) and substituting into (5.7), we arrive at the estimate

$$
K_{2} \ll\left(P^{2}(\log P)^{-150}\right)\left(\mathscr{G}_{1}(0)^{2} P^{-4}(\log P)^{14}\right) \ll \mathscr{G}_{1}(0)^{2} P^{-2}(\log P)^{-100} .
$$

Finally, on collecting together (5.5), (5.9) and (5.11), we may conclude that

$$
R(n ; \mathfrak{i}) \ll \mathscr{G}_{1}(0)^{2} P^{-2}(\log P)^{-100} .
$$

Next we investigate the contribution to $R(n)$ arising from the major arcs $\mathfrak{N}$. It transpires that this is essentially a routine exercise using the methods of Chapters 7 and 8 of Hua [8]. Write

$$
S^{*}(q, a)=\sum_{\substack{r=1 \\(r, q)=1}}^{q} e\left(a r^{4} / q\right) \quad \text { and } \quad u_{j}(\beta)=\int_{P_{j}}^{2 P_{j}} \frac{e\left(\beta t^{4}\right)}{\log t} d t \quad(1 \leqslant j \leqslant 5) .
$$

Also, let $\varphi(q)$ denote Euler's totient function. Then, as a consequence of the Siegel-Walfisz theorem, one may show that for $\alpha \in \mathfrak{N}(q, a) \subseteq \mathfrak{N}$, one has

$$
g_{j}(\alpha)=\varphi(q)^{-1} S^{*}(q, a) u_{j}(\alpha-a / q)+O\left(P_{j} L^{-5}\right) \quad(1 \leqslant j \leqslant 5)
$$

(this is essentially Lemma 7.15 of Hua [8]). Since the measure of $\mathfrak{N}$ is plainly $O\left(L^{3} P^{-4}\right)$, on substituting the above estimate into (5.1), a simple calculation reveals that

$$
R(n ; \mathfrak{N})=\subseteq(n ; L) J\left(n ; L P^{-4}\right)+O\left(\mathscr{G}_{1}(0)^{2} P^{-2} L^{-1}\right),
$$

where

$$
\Im(n ; L)=\sum_{1 \leqslant q \leqslant L} \sum_{\substack{a=1 \\(a, q)=1}}^{q}\left(\varphi(q)^{-1} S^{*}(q, a)\right)^{14} e(-a n / q)
$$


and

$$
J(n ; W)=\int_{-W}^{W} u_{1}(\beta)^{4} u_{2}(\beta)^{2} u_{3}(\beta)^{2} u_{4}(\beta)^{2} u_{5}(\beta)^{4} e(-n \beta) d \beta .
$$

In order to dispose of the singular series $\subseteq(n ; L)$, we first note that by Lemma 8.5 of [8] (see also [6]), whenever $(a, q)=1$ one has

whence

$$
S^{*}(q, a) \ll q^{1 / 2+\varepsilon},
$$

$$
\sum_{q>L} \sum_{\substack{a=1 \\(a, q)=1}}^{q}\left(\varphi(q)^{-1} S^{*}(q, a)\right)^{14} e(-a n / q) \ll \sum_{q>L} \varphi(q)^{-13} q^{7+\varepsilon} \ll L^{-4} .
$$

Then on writing

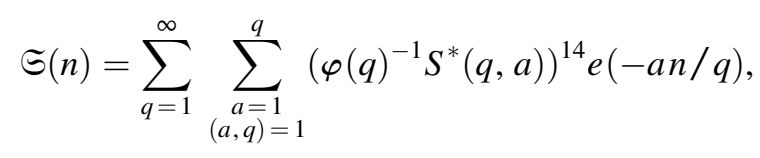

we deduce that the series $\mathfrak{S}(n)$ is absolutely convergent, and we find from (5.14) that

$$
\Im(n)-\Im(n ; L) \ll \sum_{q>L} \varphi(q)^{-13} q^{7+\varepsilon} \ll L^{-4},
$$

whence

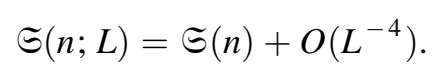

Moreover, Theorem 12 of $[8]$ assures us that

$$
\Im(n) \gg 1
$$

for all integers $n$ with $n \equiv 14(\bmod 240)$.

We begin our investigation of the singular integral $J\left(n ; L P^{-4}\right)$ by noting that a combination of a trivial estimate, together with a partial integration, yields

$$
u_{j}(\beta) \ll P_{j}\left(\log P_{j}\right)^{-1}\left(1+P_{j}^{4}|\beta|\right)^{-1} \quad(1 \leqslant j \leqslant 5) .
$$

Then on writing

$$
J(n)=\int_{-\infty}^{\infty} u_{1}(\beta)^{4} u_{2}(\beta)^{2} u_{3}(\beta)^{2} u_{4}(\beta)^{2} u_{5}(\beta)^{4} e(-n \beta) d \beta,
$$

we find that

$$
J(n) \ll(P / \log P)^{2} \mathscr{G}_{1}(0)^{2} \int_{0}^{\infty} \frac{d \beta}{\left(1+P^{4} \beta\right)^{4}} \ll P^{-2} \mathscr{G}_{1}(0)^{2}(\log P)^{-2},
$$

and from (5.15) we obtain

$$
J(n)-J\left(n ; L P^{-4}\right) \ll P^{2} \mathscr{G}_{1}(0)^{2} \int_{L P^{-4}}^{\infty} \frac{d \beta}{\left(1+P^{4} \beta\right)^{4}} \ll P^{-2} \mathscr{G}_{1}(0)^{2} L^{-3} .
$$

Since, by a change of variable,

$$
u_{j}(\beta)=\int_{P_{j}^{4}}^{\left(2 P_{j}\right)^{4}} \frac{t^{-3 / 4} e(\beta t)}{\log t} d t
$$


an application of Fourier's integral formula reveals that

$$
J(n)=\int_{\mathscr{D}} \frac{\left(\widetilde{n} t_{2} t_{3} \ldots t_{14}\right)^{-3 / 4}}{(\log \widetilde{n})\left(\log t_{2}\right)\left(\log t_{3}\right) \ldots\left(\log t_{14}\right)} d t_{2} d t_{3} \ldots d t_{14}
$$

where we write $\widetilde{n}=n-t_{2}-t_{3}-\ldots-t_{14}$, and where the region $\mathscr{D}$ of integration is the set of points $\left(t_{2}, \ldots, t_{14}\right) \in \mathbb{R}^{13}$ such that

$$
\begin{gathered}
P^{4} \leqslant \widetilde{n}, t_{2}, t_{3}, t_{4} \leqslant(2 P)^{4}, \\
P_{j}^{4} \leqslant t_{2 j+1}, t_{2 j+2} \leqslant\left(2 P_{j}\right)^{4} \quad(2 \leqslant j \leqslant 4), \\
P_{5}^{4} \leqslant t_{i} \leqslant\left(2 P_{5}\right)^{4} \quad(11 \leqslant i \leqslant 14) .
\end{gathered}
$$

Let $\mathscr{D}_{0}$ be the set of points $\left(t_{2}, \ldots, t_{14}\right) \in \mathbb{R}^{13}$ such that

$$
P^{4} \leqslant t_{2}, t_{3}, t_{4} \leqslant 2 P^{4}
$$

and the conditions (5.21) hold. On noting that whenever $\left(t_{2}, \ldots, t_{14}\right) \in \mathscr{D}_{0}$, one has $P^{4} \leqslant \widetilde{n} \leqslant(2 P)^{4}$, we find that $\mathscr{D}_{0} \subseteq \mathscr{D}$. Consequently, we deduce from (5.20) that

$$
\begin{aligned}
J(n) & \gg\left(\left(P \mathscr{F}_{1}(0)\right)^{8}\right)^{-3 / 4}(\log P)^{-14} \int_{\mathscr{D}_{0}} d t_{2} d t_{3} \ldots d t_{14} \\
& \gg\left(P \mathscr{F}_{1}(0)\right)^{-6}(\log P)^{-14} P^{4} \mathscr{F}_{1}(0)^{8} .
\end{aligned}
$$

On recalling (5.8), (5.18) and (5.19), we therefore conclude that

$$
J\left(n ; L P^{-4}\right) \asymp \mathscr{G}_{1}(0)^{2} P^{-2}(\log P)^{-2} .
$$

We now reach the crescendo of our argument for sums of fourth powers. On combining (5.13), (5.16), (5.17) and (5.22), we conclude that for every large integer $n$ with $n \equiv 14(\bmod 240)$, one has

$$
R(n ; \mathfrak{N})=\Xi(n) J\left(n ; L P^{-4}\right)+O\left(\mathscr{G}_{1}(0)^{2} P^{-2} L^{-1}\right) \gg \mathscr{G}_{1}(0)^{2} P^{-2}(\log P)^{-2} .
$$

Consequently, for each such $n$ it follows from (5.2) and (5.12) that

$$
R(n)=R(n ; \mathfrak{N})+R(n ; \mathfrak{n}) \gg \mathscr{G}_{1}(0)^{2} P^{-2}(\log P)^{-2},
$$

whence $R(n)>0$. This completes the proof of Theorem 1 .

\section{Mean value estimates for exponential sums, II}

The remainder of this paper is devoted to the proof of Theorem 2, and so henceforth we restrict attention to the case $k=5$. In this section we augment the mean value estimates of $\S 4$ with additional estimates required in the course of our deliberations. We fix notation as follows:

$$
\begin{gathered}
\lambda_{j}=\left(\frac{33}{40}\right)^{j-1} \quad(1 \leqslant j \leqslant 6), \\
\lambda_{7}=\left(\frac{33}{40}\right)^{5} \cdot \frac{136}{163}, \quad \lambda_{8}=\left(\frac{33}{40}\right)^{5} \cdot \frac{576}{815}, \quad \lambda_{9}=\left(\frac{33}{40}\right)^{5} \cdot \frac{512}{815}, \\
P_{j}=P^{\lambda_{j}}, \quad f_{j}(\alpha)=\sum_{P_{j}<x \leqslant 2 P_{j}} e\left(\alpha x^{5}\right) \quad(1 \leqslant j \leqslant 9), \\
\mathscr{F}_{j}(\alpha)=f_{9}(\alpha)^{2} \prod_{i=j}^{8} f_{i}(\alpha) \quad(1 \leqslant j \leqslant 8) .
\end{gathered}
$$


The exponents $\lambda_{j}$ result from applying the algorithm described in (3.10)-(3.12) and (3.28) of Vaughan [17] together with the theorem of Thanigasalam [15]. Thus, initiating the process with $\theta_{9}=\frac{8}{9}$ and $\alpha_{8}=\frac{5}{9}$, we define $\alpha_{j}, \nu_{j}$ and $\theta_{j}$ recursively as $j$ decreases by

$$
\begin{aligned}
& \nu_{j}= \begin{cases}\frac{4-4 \alpha_{j}}{7+\alpha_{j}}, & \text { when } j=8,7, \\
\frac{1}{8}, & \text { when } 6 \geqslant j \geqslant 2,\end{cases} \\
& \theta_{j}=\frac{1}{5}\left(4+\nu_{j}\right), \quad \alpha_{j-1}=\frac{1}{5}+\theta_{j} \alpha_{j} .
\end{aligned}
$$

We remark here that, with a modicum of computation, one finds that

$$
\alpha_{1}=\frac{83151270787}{83456000000}>1-0.0036514
$$

The values of $\lambda_{j}$ recorded in (6.1) and (6.2) are then obtained recursively by means of the relation $\lambda_{j}=\theta_{j} \lambda_{j-1}(2 \leqslant j \leqslant 9)$, starting from the initial value $\lambda_{1}=1$. This definition of the $\lambda_{j}$ leads us to the following conclusion, in view of the work of Thanigasalam [15] and Vaughan [17].

\section{LEMMA 6.1. We have}

$$
\int_{0}^{1}\left|\mathscr{F}_{j}(\alpha)\right|^{2} d \alpha \ll P^{\varepsilon} \mathscr{F}_{j}(0) \quad(1 \leqslant j \leqslant 8) .
$$

Proof. When $j=8$, the conclusion of the lemma is immediate from the theorem of Thanigasalam [15]. When $1 \leqslant j \leqslant 7$, meanwhile, one may apply the method of the proof of (3.31) of Vaughan [17] (see, in particular, the line preceding (3.20) of [17]) in order to establish recursively the desired conclusion as $j$ decreases from 7 to 1 .

We note that a conclusion slightly sharper than that presented may be established by applying the methods of Thanigasalam [14]. However, such estimates do not enhance or simplify the deliberations of this paper.

As in our discussion of fourth powers, we require some auxiliary mean value estimates.

Lemma 6.2. When $j$ is an integer with $1 \leqslant j \leqslant 9$, write

$$
I_{j}=\int_{0}^{1}\left|f_{j}(\alpha) \mathscr{F}_{1}(\alpha)\right|^{2} d \alpha .
$$

Then for $1 \leqslant j \leqslant 9$, one has

$$
I_{j} \ll \mathscr{F}_{1}(0)^{2} P_{j}^{2} P^{-5}(\log P)^{2} .
$$

Proof. We begin by recording some simple estimates, and associated notation, that facilitate our subsequent discussion. Note first that, with $\alpha_{1}$ defined as in (6.5), we find from (6.3) and (6.4) that

$$
\mathscr{F}_{1}(0) \asymp P^{5 \alpha_{1}} .
$$


Thus we find that

$$
P^{5} \mathscr{F}_{1}(0)^{-1} P_{9}^{-1 / 8} \leqslant P^{-0.0117},
$$

whence

$$
P^{5+\varepsilon} \mathscr{F}_{1}(0)^{-1} P_{j}^{-1 / 8}<1 \quad(1 \leqslant j \leqslant 9) .
$$

Next we write

$$
Y_{j}=P^{5} P_{j}^{-5} \mathscr{F}_{j}(0) \mathscr{F}_{1}(0)^{-1} \quad(2 \leqslant j \leqslant 6),
$$

and we observe that for $2 \leqslant j \leqslant 6$, one has

$$
Y_{j} \asymp \prod_{i=1}^{j-1}\left(P_{i}^{4} P_{i+1}^{-5}\right)=\prod_{i=1}^{j-1} P_{i}^{-1 / 8} .
$$

For later convenience, we also define $Z_{0}=0$, and when $1 \leqslant j \leqslant 6$ we put

$$
Z_{j}=(\log P)^{2}+P^{\varepsilon} \sum_{i=2}^{j} Y_{j} .
$$

In view of (6.8), of course, we have

$$
Z_{j} \ll(\log P)^{2} \quad(0 \leqslant j \leqslant 6) .
$$

We establish the lemma for $1 \leqslant j \leqslant 6$ following the strategy adopted in the proof of Lemma 4.4. A $(j-1)$-fold application of Lemma 4.1 to estimate $I_{j}$ reveals that for $1 \leqslant j \leqslant 6$, one has

$$
I_{j} \ll\left(\mathscr{F}_{1}(0) / \mathscr{F}_{j}(0)\right)^{1+\varepsilon} \int_{0}^{1}\left|f_{j}(\alpha) \mathscr{F}_{j}(\alpha)\right|^{2} d \alpha+\mathscr{F}_{1}(0)^{2} P_{j}^{2} P^{-5} Z_{j-1} .
$$

On applying Lemma 4.2 in combination with Lemma 6.1 to estimate the integral on the right-hand side of (6.10), we obtain for $1 \leqslant j \leqslant 6$ the upper bound

$$
\begin{aligned}
I_{j} & \ll\left(\mathscr{F}_{1}(0) / \mathscr{F}_{j}(0)\right) P_{j}^{2-1 / 8+\varepsilon} \mathscr{F}_{j}(0)+\mathscr{F}_{1}(0)^{2} P_{j}^{2} P^{-5} Z_{j} \\
& \ll \mathscr{F}_{1}(0)^{2} P_{j}^{2} P^{-5}\left(P^{5+\varepsilon} \mathscr{F}_{1}(0)^{-1} P_{j}^{-1 / 8}+Z_{j}\right) .
\end{aligned}
$$

In view of (6.7) and (6.9), therefore, we may conclude that

$$
I_{j} \ll \mathscr{F}_{1}(0)^{2} P_{j}^{2} P^{-5}(\log P)^{2} \quad(1 \leqslant j \leqslant 6),
$$

which suffices to establish the conclusion of the lemma in these cases.

When $7 \leqslant j \leqslant 9$, we apply Lemma 4.1 five times to obtain

$$
I_{j} \ll\left(\mathscr{F}_{1}(0) / \mathscr{F}_{6}(0)\right)^{1+\varepsilon} \int_{0}^{1}\left|f_{j}(\alpha) \mathscr{F}_{6}(\alpha)\right|^{2} d \alpha+\mathscr{F}_{1}(0)^{2} P_{j}^{2} P^{-5} Z_{5} .
$$

We first consider the situation in which $j=7$. Here, on applying Lemma 4.2 to the integral on the right-hand side of (6.11), taking $g(\alpha)=f_{6}(\alpha) f_{9}(\alpha)^{2}$, we obtain

$$
\begin{aligned}
\int_{0}^{1}\left|f_{7}(\alpha) \mathscr{F}_{6}(\alpha)\right|^{2} d \alpha & =\int_{0}^{1}\left|f_{7}(\alpha)^{2} f_{8}(\alpha) g(\alpha)\right|^{2} d \alpha \\
& \ll P_{7}^{2-1 / 8+\varepsilon} \int_{0}^{1}\left|\mathscr{F}_{6}(\alpha)\right|^{2} d \alpha+\mathscr{F}_{6}(0)^{2} P_{7}^{\varepsilon-3} .
\end{aligned}
$$


Applying Lemma 6.1 to the integral on the right-hand side of the last inequality, and substituting into (6.11), we deduce that

$$
I_{7} \ll \mathscr{F}_{1}(0)^{2} P_{7}^{2} P^{-5}\left(P^{5+\varepsilon} \mathscr{F}_{1}(0)^{-1} P_{7}^{-1 / 8}+P^{5+\varepsilon} P_{7}^{-5} \mathscr{F}_{6}(0) \mathscr{F}_{1}(0)^{-1}+Z_{5}\right),
$$

and hence, on recalling (6.7) and (6.9), we find that the desired bound for $j=7$ is confirmed by observing that

$$
P^{5} P_{7}^{-5} \mathscr{F}_{6}(0) \mathscr{F}_{1}(0)^{-1}=P^{4}\left(P_{2} P_{3} P_{4} P_{5}\right)^{-1} P_{7}^{-5}<P^{-0.12} \text {. }
$$

We dispose of the situations in which $j=8$ and 9 through yet another application of the Hardy-Littlewood method, and in this context we must define further Hardy-Littlewood dissections. When $j=8$ and 9 , put $Q_{j}=P_{j}^{5 / 16}$, and recalling the notation defined in (2.15) and (2.16), write

$$
\mathfrak{M}_{j}(q, a)=\mathfrak{M}\left(q, a ; Q_{j} ; P_{j}^{5}\right), \quad \mathfrak{M}_{j}=\mathfrak{M}\left(Q_{j} ; P_{j}^{5}\right), \quad \mathfrak{m}_{j}=\mathfrak{m}\left(Q_{j} ; P_{j}^{5}\right) .
$$

According to Lemma 2.1, we have

$$
\sup _{\alpha \in \mathfrak{m}_{j}}\left|f_{j}(\alpha)\right| \ll P_{j}^{1-1 / 16+\varepsilon},
$$

and also, when $\alpha \in \mathfrak{M}_{j}(q, a) \subseteq \mathfrak{M}_{j}$, one has

$$
f_{j}(\alpha) \ll w_{5}(q) P_{j} .
$$

In particular, therefore, on combining (6.13) with the conclusion of Lemma 6.1, we find that

$$
\int_{\mathfrak{m}_{j}}\left|f_{j}(\alpha) \mathscr{F}_{6}(\alpha)\right|^{2} d \alpha \ll P_{j}^{2-1 / 8+\varepsilon} \widetilde{F}_{6}(0) .
$$

Next suppose temporarily that $\alpha \in \mathfrak{M}_{j}(q, a) \subseteq \mathfrak{M}_{j}$. Observe that $P_{9}=P_{8}^{8 / 9}$, and that

$$
1 \leqslant q \leqslant Q_{j} \leqslant P_{8}^{5 / 16}, \quad|q \alpha-a| \leqslant Q_{j} P_{j}^{-5} \leqslant P_{9}^{-75 / 16}=P_{8}^{-25 / 6} .
$$

Then on recalling (2.2) we find that

$$
\frac{w_{5}(q) P_{8}}{1+P_{8}^{5}|\alpha-a / q|} \geqslant q^{1 / 2} \frac{P_{8}}{q+P_{8}^{5}|q \alpha-a|} \gg q^{1 / 2+\varepsilon},
$$

whence by Lemmata 6.1 and 6.2 of [19], one has

$$
f_{8}(\alpha) \ll \frac{w_{5}(q) P_{8}}{1+P_{8}^{5}|\alpha-a / q|}+q^{1 / 2+\varepsilon} \ll \frac{w_{5}(q) P_{8}}{1+P_{8}^{5}|\alpha-a / q|} .
$$

By a similar argument, we obtain

$$
f_{9}(\alpha) \ll w_{5}(q) P_{9}+q^{1 / 2+\varepsilon} \ll w_{5}(q) P_{9} .
$$

Furthermore, again applying Lemmata 6.1 and 6.2 of Vaughan [19] in the sharper form permitted by the proof of Theorem 4.1 of [19], we have

$$
\begin{aligned}
f_{7}(\alpha) & \ll \frac{w_{5}(q) P_{7}}{1+P_{7}^{5}|\alpha-a / q|}+q^{1 / 2+\varepsilon}\left(1+P_{7}^{5}|\alpha-a / q|\right)^{1 / 2} \\
& \ll \frac{w_{5}(q) P_{7}}{1+P_{7}^{5}|\alpha-a / q|}+q^{1 / 2+\varepsilon}\left(1+P_{8}^{5}|\alpha-a / q|\right)^{1 / 2}\left(P_{7} / P_{8}\right)^{5 / 2} .
\end{aligned}
$$


Combining the bounds (6.14) and (6.16)-(6.18), together with the trivial bound $f_{6}(\alpha)=O\left(P_{6}\right)$, we deduce that

$$
\int_{\mathfrak{M}_{j}}\left|f_{j}(\alpha) \mathscr{F}_{6}(\alpha)\right|^{2} d \alpha \ll I_{j}^{\prime}+I_{j}^{\prime \prime},
$$

where

$$
I_{j}^{\prime}=P_{j}^{2} \mathscr{F}_{6}(0)^{2} \sum_{1 \leqslant q \leqslant Q_{j}} q w_{5}(q)^{10} \int_{0}^{\infty} \frac{d \beta}{\left(1+P_{7}^{5} \beta\right)^{2}},
$$

and

$$
I_{j}^{\prime \prime}=P_{j}^{2} P_{6}^{2} P_{8}^{2} P_{9}^{4}\left(P_{7} / P_{8}\right)^{5} \sum_{1 \leqslant q \leqslant Q_{j}} q^{2+\varepsilon} w_{5}(q)^{8} \int_{0}^{1} \frac{d \beta}{1+P_{8}^{5} \beta} .
$$

Applying Lemma 2.4 within (6.20), we find that

$$
I_{j}^{\prime} \ll P_{j}^{2} \mathscr{F}_{6}(0)^{2} P_{7}^{-5} \text {. }
$$

Meanwhile, also by Lemma 2.4,

$$
\sum_{1 \leqslant q \leqslant Q_{j}} q^{2+\varepsilon} w_{5}(q)^{8} \ll Q_{j}^{3 / 5+\varepsilon} \sum_{1 \leqslant q \leqslant Q_{j}} q^{7 / 5} w_{5}(q)^{8} \ll P_{8}^{3 / 16+\varepsilon},
$$

whence by (6.21), on noting also that $P_{8}=P_{7}^{72 / 85}$, we have

$$
I_{j}^{\prime \prime} \ll P_{j}^{2} \mathscr{F}_{6}(0)^{2} P_{7}^{3} P_{8}^{\varepsilon-10+3 / 16} \ll P_{j}^{2} \mathscr{F}_{6}(0)^{2} P_{7}^{-5} .
$$

On combining (6.15), (6.19), (6.22) and (6.23), we arrive at the upper bound

$$
\begin{aligned}
\int_{0}^{1}\left|f_{j}(\alpha) \mathscr{F}_{6}(\alpha)\right|^{2} d \alpha & =\int_{\mathfrak{m}_{j}}\left|f_{j}(\alpha) \mathscr{F}_{6}(\alpha)\right|^{2} d \alpha+\int_{\mathfrak{M}_{j}}\left|f_{j}(\alpha) \mathscr{F}_{6}(\alpha)\right|^{2} d \alpha \\
& \ll P_{j}^{2-1 / 8+\varepsilon} \mathscr{F}_{6}(0)+P_{j}^{2} \mathscr{F}_{6}(0)^{2} P_{7}^{-5},
\end{aligned}
$$

valid for $j=8$ and 9. But on recalling (6.11), we see that the latter bound implies that

$$
I_{j} \ll \mathscr{F}_{1}(0)^{2} P_{j}^{2} P^{-5}\left(P^{5+\varepsilon} \mathscr{F}_{1}(0)^{-1} P_{j}^{-1 / 8}+P^{5+\varepsilon} P_{7}^{-5} \mathscr{F}_{6}(0) \mathscr{F}_{1}(0)^{-1}+Z_{5}\right) .
$$

Hence the desired bounds for $j=8$ and 9 follow by virtue of the inequalities (6.7), (6.9) and (6.12).

This completes the proof of the lemma.

We may now prepare the key minor arc estimate, but before announcing this result we require some notation. We define

$$
g_{j}(\alpha)=\sum_{P_{j}<p \leqslant 2 P_{j}} e\left(p^{5} \alpha\right) \quad(1 \leqslant j \leqslant 9)
$$

and then write

$$
\mathscr{G}_{2}(\alpha)=g_{9}(\alpha)^{2} \prod_{i=2}^{8} g_{i}(\alpha)
$$


We define our primary Hardy-Littlewood dissection as follows. We write $L=(\log P)^{10000}$, and define $\mathfrak{N}$ to be the union of the arcs

$$
\mathfrak{N}(q, a)=\left\{\alpha \in[0,1):|\alpha-a / q| \leqslant L P^{-5}\right\},
$$

with $0 \leqslant a \leqslant q \leqslant L$ and $(a, q)=1$. We then put $\mathfrak{n}=[0,1) \backslash \mathfrak{N}$.

Lemma 6.3. Let $H(\alpha)$ be a complex-valued function of $\alpha$, periodic with period 1. Suppose that $H(\alpha)$ has the property that whenever $\alpha$ is a real number, and $a \in \mathbb{Z}$ and $q \in \mathbb{N}$ satisfy

$$
(a, q)=1, \quad 1 \leqslant q \leqslant P^{5 / 2} \text { and }|q \alpha-a| \leqslant P^{-5 / 2},
$$

then one has

$$
H(\alpha) \ll P^{1-c+\varepsilon}+\frac{q^{\varepsilon} w_{5}(q)^{1 / 2} P(\log P)^{5}}{\left(1+P^{5}|\alpha-a / q|\right)^{1 / 2}},
$$

with $c=0.0183$. Suppose also that $\mathscr{H} \subseteq(P, 2 P] \cap \mathbb{Z}$, and write

$$
h(\alpha)=\sum_{x \in \mathscr{H}} e\left(\alpha x^{5}\right) \text {. }
$$

Then we have

$$
\int_{\mathfrak{n}}\left|H(\alpha) h(\alpha) g_{1}(\alpha) \mathscr{G}_{2}(\alpha)^{2}\right| d \alpha \ll \mathscr{G}_{2}(0)^{2} P^{-2}(\log P)^{-100}
$$

We remark that the conclusion of the lemma remains valid for any $c$ satisfying $c>5-5 \alpha_{1}$, where $\alpha_{1}$ is given by (6.5).

The proof of Lemma 6.3. We apply a pruning procedure, and hence require a further Hardy-Littlewood dissection. Recalling the notation defined in (2.15) and (2.16), we write

$$
\mathfrak{M}(q, a)=\mathfrak{M}\left(q, a ; P^{10 c} ; P^{5}\right), \quad \mathfrak{M}=\mathfrak{M}\left(P^{10 c} ; P^{5}\right), \quad \mathrm{m}=\mathfrak{m}\left(P^{10 c} ; P^{5}\right) .
$$

Plainly, one has $\mathrm{m} \subseteq \mathfrak{n}$.

We define the function $\Psi(\alpha)$ for $\alpha \in \mathfrak{M}$ by taking

$$
\Psi(\alpha)=q^{\delta} w_{5}(q)\left(1+P^{5}|\alpha-a / q|\right)^{-1},
$$

when $\alpha \in \mathfrak{M}(q, a) \subseteq \mathfrak{M}$, where we write $\delta=10^{-2}$. In view of (2.2), whenever $\alpha \in \mathfrak{M}(q, a) \subseteq \mathfrak{M}$ and $\alpha \in \mathfrak{n}$, one has

$$
\Psi(\alpha) \ll\left(q+P^{5}|q \alpha-a|\right)^{\delta-1 / 5} \ll L^{-1 / 10},
$$

and thus our hypotheses concerning the function $H(\alpha)$ ensure that for $\alpha \in \mathfrak{M} \cap \mathfrak{n}$, one has

$$
\begin{aligned}
H(\alpha) & \ll P^{1-c+\varepsilon}+P(\log P)^{5} \Psi(\alpha)^{1 / 2} \\
& \ll P^{1-c+\varepsilon}+P(\log P)^{5} L^{-1 / 50} \Psi(\alpha)^{3 / 10} .
\end{aligned}
$$

Meanwhile, when $\alpha \in \mathfrak{m}$, we may apply Dirichlet's approximation theorem to deduce the existence of $a \in \mathbb{Z}$ and $q \in \mathbb{N}$ satisfying the conditions (6.26), and necessarily one has either

$$
q>P^{10 c} \text { or }|q \alpha-a|>P^{10 c-5} .
$$


In this situation it follows from (2.2) that

$$
\frac{w_{5}(q)^{1 / 2} P}{\left(1+P^{5}|\alpha-a / q|\right)^{1 / 2}} \ll \frac{P}{\left(q+P^{5}|q \alpha-a|\right)^{1 / 10}} \ll P^{1-c},
$$

and so our hypotheses on $H(\alpha)$ ensure that

$$
\sup _{\alpha \in \mathfrak{m}}|H(\alpha)| \ll P^{1-c+\varepsilon} .
$$

On exploiting the estimates (6.27) and (6.28) within the mean value under consideration, we deduce that

$$
\int_{\mathfrak{n}}\left|H(\alpha) h(\alpha) g_{1}(\alpha) \mathscr{G}_{2}(\alpha)^{2}\right| d \alpha \ll K_{1}+K_{2},
$$

where

$$
K_{1}=P^{1-c+\varepsilon} \int_{0}^{1}\left|h(\alpha) g_{1}(\alpha) \mathscr{G}_{2}(\alpha)^{2}\right| d \alpha
$$

and

$$
K_{2}=P(\log P)^{-150} \int_{\mathfrak{M} \cap \mathfrak{n}} \Psi(\alpha)^{3 / 10}\left|h(\alpha) g_{1}(\alpha) \mathscr{G}_{2}(\alpha)^{2}\right| d \alpha .
$$

We first estimate $K_{1}$, noting that by considering the underlying diophantine equations, it follows from Lemma 6.1 that

$$
\int_{0}^{1}\left|h(\alpha) \mathscr{G}_{2}(\alpha)\right|^{2} d \alpha \ll \int_{0}^{1}\left|\mathscr{F}_{1}(\alpha)\right|^{2} d \alpha \ll P^{\varepsilon} \mathscr{F}_{1}(0) .
$$

Similarly, one has also

$$
\int_{0}^{1}\left|g_{1}(\alpha) \mathscr{G}_{2}(\alpha)\right|^{2} d \alpha \ll \int_{0}^{1}\left|\mathscr{F}_{1}(\alpha)\right|^{2} d \alpha \ll P^{\varepsilon} \mathscr{F}_{1}(0),
$$

and thus an application of Schwarz's inequality yields the upper bound

$$
\begin{aligned}
\int_{0}^{1}\left|h(\alpha) g_{1}(\alpha) \mathscr{G}_{2}(\alpha)^{2}\right| d \alpha & \ll\left(\int_{0}^{1}\left|h(\alpha) \mathscr{G}_{2}(\alpha)\right|^{2} d \alpha\right)^{1 / 2} \\
& \times\left(\int_{0}^{1}\left|g_{1}(\alpha) \mathscr{G}_{2}(\alpha)\right|^{2} d \alpha\right)^{1 / 2} \\
& \ll P^{\varepsilon} \mathscr{F}_{1}(0) .
\end{aligned}
$$

But a comparison of (6.3), (6.4) and (6.24), (6.25) reveals that

$$
\mathscr{G}_{2}(0) \asymp \mathscr{F}_{1}(0) P^{-1}(\log P)^{-9} \text {. }
$$

Then on recalling (6.5) and (6.6), we deduce from (6.30) that

$$
K_{1} \ll \mathscr{G}_{2}(0)^{2} P^{\varepsilon-2}\left(P^{5-c} \mathscr{F}_{1}(0)^{-1}\right) \ll \mathscr{G}_{2}(0)^{2} P^{-2.00004}
$$

Now we turn our attention to the estimation of $K_{2}$. By Hölder's inequality, one has

$$
\int_{\mathfrak{M}} \Psi(\alpha)^{3 / 10}\left|h(\alpha) g_{1}(\alpha) \mathscr{G}_{2}(\alpha)^{2}\right| d \alpha \ll K_{3}^{1 / 10} \prod_{j=1}^{9} J_{j}^{1 / 10},
$$


where

and for $1 \leqslant j \leqslant 5$,

$$
K_{3}=\int_{\mathfrak{M}} \Psi(\alpha)^{3}\left|g_{9}(\alpha)\right|^{2} d \alpha
$$

while for $6 \leqslant j \leqslant 9$,

$$
J_{j}=\int_{0}^{1}\left|g_{j}(\alpha) h(\alpha) \mathscr{G}_{2}(\alpha)\right|^{2} d \alpha,
$$

$$
J_{j}=\int_{0}^{1}\left|g_{j}(\alpha) g_{1}(\alpha) \mathscr{G}_{2}(\alpha)\right|^{2} d \alpha .
$$

But by considering the underlying diophantine equations, we deduce from Lemma 6.2 that

$$
J_{j} \leqslant I_{j} \ll \mathscr{F}_{1}(0)^{2} P_{j}^{2} P^{-5}(\log P)^{2} \quad(1 \leqslant j \leqslant 9) .
$$

Meanwhile, since $P^{10 c}<P_{9}$, we find that Lemma 2.5 supplies the bound

$$
\int_{\mathfrak{M}} \Psi(\alpha)^{3}\left|g_{9}(\alpha)\right|^{2} d \alpha \ll P_{9}^{2} P^{-5}
$$

Thus we may conclude from (6.32) and (6.34) that

$$
\begin{aligned}
\int_{\mathfrak{M}} \Psi(\alpha)^{3 / 10}\left|h(\alpha) g_{1}(\alpha) \mathscr{G}_{2}(\alpha)^{2}\right| d \alpha & \ll \mathscr{F}_{1}(0)^{2} P^{-5}(\log P)^{2} \\
& \ll \mathscr{G}_{2}(0)^{2} P^{-3}(\log P)^{20},
\end{aligned}
$$

whence by $(6.31)$ we arrive at the estimate

$$
K_{2} \ll \mathscr{G}_{2}(0)^{2} P^{-2}(\log P)^{-100} .
$$

The conclusion of the lemma is established by combining (6.29), (6.33) and (6.35).

\section{The Waring-Goldbach problem for fifth powers}

In this section we complete the bulk of the work associated with the proof of Theorem 2, retaining the notation introduced in the previous section. We note first that in order to establish that $H(5) \leqslant 21$, it suffices to show that all sufficiently large odd numbers are the sum of 21 fifth powers of prime numbers. For when $s$ is a natural number with $s \geqslant 21$, and $n$ is a sufficiently large integer with $n \equiv s$ $(\bmod 2)$, then $n_{1}=n-(s-21) \cdot 3^{5}$ satisfies $n_{1} \equiv 1(\bmod 2)$. Consequently, $n$ is represented as the sum of $s$ fifth powers of prime numbers whenever $n_{1}$ is the sum of 21 fifth powers of prime numbers. Suppose temporarily that all sufficiently large odd numbers are indeed the sum of 21 fifth powers of prime numbers. Then it follows that whenever $s \geqslant 22$, then all sufficiently large integers are the sum of $s$ fifth powers of prime numbers. For when $s \geqslant 22$ and $n$ is a sufficiently large natural number, then one of $n-(s-21) \cdot 3^{5}$ and $n-(s-22) \cdot 3^{5}-2^{5}$ is odd, and hence equal to the sum of 21 fifth powers of prime numbers.

Consider then a sufficiently large odd natural number $n$, and write

$$
P=\frac{1}{2} n^{1 / 5} \text {. }
$$

When $\mathfrak{X} \subseteq[P, 2 P] \cap \mathbb{Z}$, we denote by $R(n, \mathfrak{X})$ the number of representations of 
the natural number $n$ in the form

$$
n=x^{5}+p_{1}^{5}+p_{2}^{5}+\ldots+p_{20}^{5},
$$

with

$$
\begin{gathered}
x \in \mathfrak{X}, \quad P_{j}<p_{2 j-1}, p_{2 j} \leqslant 2 P_{j}(1 \leqslant j \leqslant 8), \\
P_{9}<p_{i} \leqslant 2 P_{9}(17 \leqslant i \leqslant 20) .
\end{gathered}
$$

Write $\mathfrak{X}_{0}$ for the set of prime numbers $p$ with $P<p<2 P$. Our aim is to establish Theorem 2 by showing that $R\left(n, \mathfrak{X}_{0}\right)>0$.

Next put

$$
z=(2 P)^{1 / 3} \quad \text { and } \quad z_{1}=(2 P)^{1-32 c},
$$

where $c=0.0183$ is the constant arising in the statement of Lemma 6.3. Define also

$$
\Pi(z)=\prod_{p<z} p
$$

We introduce the sets of integers

$$
\begin{aligned}
& \mathfrak{X}_{1}=\{P<x<2 P:(x, \Pi(z))=1\}, \\
& \mathfrak{X}_{2}=\left\{P<x<2 P: x=\varpi_{1} \varpi_{2} \text { and } z \leqslant \varpi_{1}<z_{1}\right\}, \\
& \mathfrak{X}_{3}=\left\{P<x<2 P: x=\varpi_{1} \varpi_{2} \text { and } z_{1} \leqslant \varpi_{1} \leqslant \varpi_{2}\right\},
\end{aligned}
$$

where here, and hereafter, we employ the letter $\varpi$ with subscripts to denote a prime number. On noting that no natural number smaller than $2 P$ can be a product of more than two primes greater than or equal to $z$, it is apparent that

$$
\mathfrak{X}_{1}=\mathfrak{X}_{0} \cup \mathfrak{X}_{2} \cup \mathfrak{X}_{3} .
$$

Since $\mathfrak{X}_{0}, \mathfrak{X}_{2}$ and $\mathfrak{X}_{3}$ are pairwise disjoint, we conclude that

$$
R\left(n, \mathfrak{X}_{0}\right)=R\left(n, \mathfrak{X}_{1}\right)-R\left(n, \mathfrak{X}_{2}\right)-R\left(n, \mathfrak{X}_{3}\right) .
$$

We first investigate $R\left(n, \mathfrak{X}_{2}\right)$. Write

$$
h_{1}(\alpha)=\sum_{x \in \mathfrak{X}_{2}} e\left(\alpha x^{5}\right),
$$

and when $\mathfrak{B} \subseteq[0,1)$, define

$$
R\left(n, \mathfrak{X}_{2} ; \mathfrak{B}\right)=\int_{\mathfrak{B}} h_{1}(\alpha) g_{1}(\alpha)^{2} \mathscr{G}_{2}(\alpha)^{2} e(-n \alpha) d \alpha .
$$

Then by orthogonality,

$$
R\left(n, \mathfrak{X}_{2}\right)=R\left(n, \mathfrak{X}_{2} ;[0,1)\right)=R\left(n, \mathfrak{X}_{2} ; \mathfrak{N}\right)+R\left(n, \mathfrak{X}_{2} ; \mathfrak{n}\right) .
$$

We are able to dispose of the contribution to $R\left(n, \mathfrak{X}_{2}\right)$ arising from $\mathfrak{n}$ almost immediately. Suppose that $\alpha$ is a real number, and that $a \in \mathbb{Z}$ and $q \in \mathbb{N}$ satisfy (6.26). Then by means of a familiar dyadic dissection argument, it follows from 
Lemma 3.1 that

$$
\begin{aligned}
h_{1}(\alpha) & =\sum_{P / z_{1}<\sigma_{2}<2 P / z} \sum_{\substack{P / \varpi_{2}<\sigma_{1}<2 P / \varpi_{2} \\
z \leqslant \sigma_{1}<z_{1}}} e\left(\left(\boldsymbol{\varpi}_{1} \boldsymbol{\varpi}_{2}\right)^{5} \alpha\right) \\
& \ll(\log P) \sup _{P / z_{1} \leqslant M \leqslant P / z}\left(P M^{\varepsilon-1 / 32}+(P M)^{1 / 2+\varepsilon}+\frac{q^{\varepsilon} w_{5}(q)^{1 / 2} P(\log P)^{4}}{\left(1+P^{5}|\alpha-a / q|\right)^{1 / 2}}\right) \\
& \ll P^{1-c+\varepsilon}+\frac{q^{\varepsilon} w_{5}(q)^{1 / 2} P(\log P)^{5}}{\left(1+P^{5}|\alpha-a / q|\right)^{1 / 2}} .
\end{aligned}
$$

It follows that $h_{1}(\alpha)$ satisfies the hypotheses imposed on the function $H(\alpha)$ in the statement of Lemma 6.3, and consequently we deduce from that lemma that

$$
R\left(n, \mathfrak{X}_{2} ; \mathfrak{n}\right) \leqslant \int_{\mathfrak{n}}\left|h_{1}(\alpha) g_{1}(\alpha)^{2} \mathscr{G}_{2}(\alpha)^{2}\right| d \alpha \ll \mathscr{G}_{2}(0)^{2} P^{-2}(\log P)^{-100} .
$$

The estimation of the contribution of the major arcs $\mathfrak{N}$ to $R\left(n, \mathfrak{X}_{2}\right)$, while routine, requires some preparation and associated notation. Write

$$
S^{*}(q, a)=\sum_{\substack{r=1 \\(r, q)=1}}^{q} e\left(a r^{5} / q\right) \quad \text { and } \quad u_{j}(\beta)=\int_{P_{j}}^{2 P_{j}} \frac{e\left(\beta t^{5}\right)}{\log t} d t \quad(1 \leqslant j \leqslant 9) .
$$

Then as a consequence of the Siegel-Walfisz theorem (see Lemma 7.15 of Hua [8]), one has, for $\alpha \in \mathfrak{N}(q, a) \subseteq \mathfrak{N}$,

$$
g_{j}(\alpha)=\varphi(q)^{-1} S^{*}(q, a) u_{j}(\alpha-a / q)+O\left(P_{j} L^{-5}\right) \quad(1 \leqslant j \leqslant 9) .
$$

The estimation of $h_{1}(\alpha)$ for $\alpha \in \mathfrak{N}$ is best accommodated within a technical lemma.

Lemma 7.1. Suppose that $\eta_{1}$ and $\eta_{2}$ are real numbers with $0<\eta_{1}<\eta_{2} \leqslant \frac{1}{2}$. Write $v_{j}=(2 P)^{\eta_{j}}(j=1,2)$, and define

where

$$
u\left(\beta ; \eta_{1}, \eta_{2}\right)=\int_{P}^{2 P} \Xi\left(t ; \eta_{1}, \eta_{2}\right) \frac{e\left(\beta t^{5}\right)}{\log t} d t,
$$

$$
\Xi\left(t ; \eta_{1}, \eta_{2}\right)=\sum_{\substack{v_{1} \leqslant \varpi_{1}<v_{2} \\ \varpi_{1} \leqslant \sqrt{t}}} \frac{\log t}{\varpi_{1} \log \left(t / \varpi_{1}\right)} .
$$

Suppose further that $\alpha$ is a real number with $\alpha=\beta+a / q$, where $(a, q)=1$, $1 \leqslant q \leqslant L$ and $|\beta| \leqslant L P^{-5}$. Then one has

$$
\begin{aligned}
& \sum_{v_{1} \leqslant \varpi_{1}<v_{2}} \sum_{\substack{P / \varpi_{1}<\varpi_{2}<2 P / \varpi_{1} \\
\varpi_{2} \geq \varpi_{1}}} e\left(\left(\varpi_{1} \varpi_{2}\right)^{5} \alpha\right) \\
&=\varphi(q)^{-1} S^{*}(q, a) u\left(\beta ; \eta_{1}, \eta_{2}\right)+O\left(P L^{-5}\right) .
\end{aligned}
$$

Moreover, with $u_{1}(\beta)$ defined as in (7.7), one has

$$
u\left(\beta ; \eta_{1}, \eta_{2}\right)=\log \left(\frac{\eta_{1}^{-1}-1}{\eta_{2}^{-1}-1}\right) u_{1}(\beta)+O\left(P(\log P)^{-2}\right) .
$$


Proof. For a pair of primes $\left(\varpi_{1}, \varpi_{2}\right)$ occurring in the summation of (7.9), our hypotheses on $v_{1}$ and $v_{2}$ ensure that $\varpi_{1}<\sqrt{2 P}$, whence $\varpi_{2}>\sqrt{P / 2}$. Thus, with an argument similar to that leading to (7.8), we deduce that

$$
\sum_{\substack{P / \varpi_{1}<\varpi_{2}<2 P / \varpi_{1} \\ \varpi_{2} \geqslant \varpi_{1}}} e\left(\left(\varpi_{1} \varpi_{2}\right)^{5} \alpha\right)=T+O\left(P \varpi_{1}^{-1} L^{-6}\right),
$$

where

$$
T=\varphi(q)^{-1} S^{*}\left(q, a \varpi_{1}^{5}\right) \int_{\max \left\{P / \varpi_{1}, \varpi_{1}\right\}}^{2 P / \varpi_{1}} \frac{e\left(u^{5} \varpi_{1}^{5} \beta\right)}{\log u} d u .
$$

On noting that $q \leqslant L<v_{1} \leqslant \varpi_{1}$, we find that $\left(q, \varpi_{1}\right)=1$, and thus a multiplicative change of variables reveals that $S^{*}\left(q, a \varpi_{1}^{5}\right)=S^{*}(q, a)$. Employing next the change of variables $t=\varpi_{1} u$ within the integral on the right-hand side of (7.12), we find that

$$
T=\varphi(q)^{-1} S^{*}(q, a) \int_{\max \left\{P, \varpi_{1}^{2}\right\}}^{2 P} \frac{e\left(\beta t^{5}\right)}{\varpi_{1} \log \left(t / \varpi_{1}\right)} d t .
$$

The formula (7.9) follows directly from (7.11) and (7.13) by summing over $\varpi_{1}$.

In order to confirm the estimate (7.10), we exploit well-known prime number estimates via partial summation to obtain

$$
\Xi\left(t ; \eta_{1}, \eta_{2}\right)=\int_{v_{1}}^{\min \left\{v_{2}, \sqrt{t}\right\}} \frac{1}{u \log u} \cdot \frac{\log t}{\log t-\log u} d u+O\left((\log t)^{-1}\right),
$$

valid for $P \leqslant t \leqslant 2 P$. But the change of variable $v=(\log t) /(\log u)$ leads to the formula

where

$$
\int_{v_{1}}^{\min \left\{v_{2}, \sqrt{t}\right\}} \frac{1}{u \log u} \cdot \frac{\log t}{\log t-\log u} d u=\int_{\sigma}^{\tau} \frac{d v}{v-1},
$$

$$
\sigma=\max \left\{\frac{\log t}{\log v_{2}}, 2\right\} \quad \text { and } \quad \tau=\frac{\log t}{\log v_{1}} .
$$

Thus we deduce from (7.14) that for $P \leqslant t \leqslant 2 P$, whenever $\eta_{2} \leqslant \frac{1}{2}$ one has

$$
\Xi\left(t ; \eta_{1}, \eta_{2}\right)=\log \left(\frac{\eta_{1}^{-1}-1}{\eta_{2}^{-1}-1}\right)+O\left((\log P)^{-1}\right) .
$$

The desired conclusion now follows immediately from (7.7) and the definition of $u\left(\beta ; \eta_{1}, \eta_{2}\right)$, and this completes the proof of the lemma.

We now return to the task of evaluating $R\left(n, \mathfrak{X}_{2} ; \mathfrak{N}\right)$. When $\alpha \in \mathfrak{N}(q, a) \subseteq \mathfrak{N}$, an application of Lemma 7.1 reveals that

$$
h_{1}(\alpha)=\varphi(q)^{-1} S^{*}(q, a) u\left(\alpha-a / q ; \frac{1}{3}, 1-32 c\right)+O\left(P L^{-5}\right) .
$$

Since the measure of $\mathfrak{N}$ is plainly $O\left(L^{3} P^{-5}\right)$, we deduce from (7.8) and (7.15) that

$$
\begin{aligned}
R\left(n, \mathfrak{X}_{2} ; \mathfrak{N}\right) & =\int_{\mathfrak{N}} h_{1}(\alpha) g_{1}(\alpha)^{2} \mathscr{G}_{2}(\alpha)^{2} e(-n \alpha) d \alpha \\
& =\subseteq(n ; L) J_{1}\left(n ; L P^{-5}\right)+O\left(\mathscr{G}_{2}(0)^{2} P^{-2} L^{-1}\right),
\end{aligned}
$$


where

$$
\Im(n ; L)=\sum_{1 \leqslant q \leqslant L} \sum_{\substack{a=1 \\(a, q)=1}}^{q}\left(\varphi(q)^{-1} S^{*}(q, a)\right)^{21} e(-a n / q)
$$

and

$$
J_{1}(n ; W)=\int_{-W}^{W} u\left(\beta ; \frac{1}{3}, 1-32 c\right) u_{1}(\beta)^{2} \mathscr{U}_{2}(\beta)^{2} e(-n \beta) d \beta,
$$

in which we have written

$$
\mathscr{U}_{2}(\beta)=u_{9}(\beta)^{2} \prod_{i=2}^{8} u_{i}(\beta) .
$$

The singular series $\subseteq(n ; L)$ is easily computed by noting that Lemma 8.5 of Hua [8] shows that when $(a, q)=1$, one has the bound

$$
S^{*}(q, a) \ll q^{1 / 2+\varepsilon},
$$

whence

$$
\sum_{q>L} \sum_{\substack{a=1 \\(a, q)=1}}^{q}\left(\varphi(q)^{-1} S^{*}(q, a)\right)^{21} e(-a n / q) \ll \sum_{q>L} \varphi(q)^{-20} q^{21 / 2+\varepsilon} \ll L^{-8} .
$$

On writing

$$
\mathfrak{S}(n)=\sum_{q=1}^{\infty} \sum_{\substack{a=1 \\(a, q)=1}}^{q}\left(\varphi(q)^{-1} S^{*}(q, a)\right)^{21} e(-a n / q),
$$

we therefore deduce that the series $\subseteq(n)$ is absolutely convergent, and from (7.17) we find that

$$
\mathfrak{S}(n ; L)=\mathfrak{S}(n)+O\left(L^{-8}\right) .
$$

Moreover, Theorem 12 of [8] guarantees that

for all odd integers $n$.

$$
\Im(n) \gg 1
$$

Turning our attention now to the evaluation of the singular integral, we define

$$
J(n)=\int_{-\infty}^{\infty} u_{1}(\beta)^{3} \mathscr{U}_{2}(\beta)^{2} e(-n \beta) d \beta .
$$

The estimate

$$
u_{j}(\beta) \ll P_{j}\left(\log P_{j}\right)^{-1}\left(1+P_{j}^{5}|\beta|\right)^{-1} \quad(1 \leqslant j \leqslant 9)
$$

is readily obtained through partial integration, and thereby we deduce that

$$
\begin{aligned}
J(n)-\int_{-L P^{-5}}^{L P^{-5}} u_{1}(\beta)^{3} \mathscr{U}_{2}(\beta)^{2} e(-n \beta) d \beta \\
\ll P^{3}(\log P)^{-3} \mathscr{U}_{2}(0)^{2} \int_{L P^{-5}}^{\infty}\left(1+P^{5} \beta\right)^{-3} d \beta \\
\ll P^{-2}(\log P)^{-3} \mathscr{U}_{2}(0)^{2} L^{-2}
\end{aligned}
$$


and

$$
\begin{aligned}
\int_{-L P^{-5}}^{L P^{-5}}\left|u_{1}(\beta) \mathscr{U}_{2}(\beta)\right|^{2} d \beta & \ll P^{2}(\log P)^{-2} \mathscr{U}_{2}(0)^{2} \int_{0}^{\infty}\left(1+P^{5} \beta\right)^{-2} d \beta \\
& \ll P^{-3}(\log P)^{-2} \mathscr{U}_{2}(0)^{2} .
\end{aligned}
$$

On recalling the conclusion of Lemma 7.1, we deduce from (7.18) that

$$
\begin{aligned}
J_{1}\left(n ; L P^{-5}\right)= & \log \left(\frac{1-32 c}{16 c}\right) \int_{-L P^{-5}}^{L P^{-5}} u_{1}(\beta)^{3} \mathscr{U}_{2}(\beta)^{2} e(-n \beta) d \beta \\
& +O\left(P(\log P)^{-2} \int_{-L P^{-5}}^{L P^{-5}}\left|u_{1}(\beta) \mathscr{U}_{2}(\beta)\right|^{2} d \beta\right),
\end{aligned}
$$

whence by (7.26) and (7.27),

$$
\begin{aligned}
J_{1}\left(n ; L P^{-5}\right)= & \log \left(\frac{1-32 c}{16 c}\right)\left(J(n)+O\left(P^{-2}(\log P)^{-3} \mathscr{U}_{2}(0)^{2} L^{-2}\right)\right) \\
& +O\left(P^{-2}(\log P)^{-4} \mathscr{U}_{2}(0)^{2}\right) .
\end{aligned}
$$

Thus we conclude that

$$
J_{1}\left(n ; L P^{-5}\right)=\log \left(\frac{1-32 c}{16 c}\right) J(n)+O\left(\mathscr{G}_{2}(0)^{2} P^{-2}(\log P)^{-4}\right) .
$$

Moreover, as in the argument leading to (5.22) above, an application of Fourier's integral formula demonstrates that

$$
J(n) \asymp \mathscr{G}_{2}(0)^{2} P^{-2}(\log P)^{-3} .
$$

We summarise our deliberations thus far in the shape of the following lemma.

Lemma 7.2. For each large odd integer $n$, one has

$$
R\left(n, \mathfrak{X}_{2}\right)=\Xi(n) J(n)\left(\log \left(\frac{1-32 c}{16 c}\right)+O\left((\log P)^{-1}\right)\right),
$$

where $J(n)$ and $\mathfrak{S}(n)$ satisfy the lower bounds

$$
J(n) \gg \mathscr{G}_{2}(0)^{2} P^{-2}(\log P)^{-3} \text { and } \subseteq(n) \gg 1 .
$$

Proof. The lemma is immediate on collecting together (7.5), (7.6), (7.16), (7.22), (7.28) with (7.23) and (7.29).

The estimation of $R\left(n, \mathfrak{X}_{1}\right)$ and $R\left(n, \mathfrak{X}_{3}\right)$ is accomplished by means of Iwaniec's linear sieve. We announce our conclusions in the imminent lemma, but defer discussion of the proof to $\S 9$.

LEMma 7.3. For each large odd integer $n$, one has

and

$$
R\left(n, \mathfrak{X}_{1}\right)>\Im(n) J(n)\left(\frac{4}{3-66 c} \log \left(\frac{7}{2}-99 c\right)+O\left((\log \log P)^{-1 / 50}\right)\right),
$$

$$
R\left(n, \mathfrak{X}_{3}\right)<\Xi(n) J(n)\left(\frac{4}{3-66 c} \log \left(\frac{32 c}{1-32 c}\right)+O\left((\log \log P)^{-1 / 50}\right)\right) .
$$


Temporarily taking as granted the conclusion of Lemma 7.3, we may complete the proof of Theorem 2. We merely observe that by combining (7.4) and the conclusions of Lemmata 7.2 and 7.3, we obtain for each odd integer $n$ the lower bound

$$
R\left(n, \mathfrak{X}_{0}\right)>\Im(n) J(n)\left(C+O\left((\log \log P)^{-1 / 50}\right)\right),
$$

where

$$
C=\frac{4}{3-66 c} \log \left(\frac{(7-198 c)(1-32 c)}{64 c}\right)-\log \left(\frac{1-32 c}{16 c}\right) .
$$

Since $c=0.0183$, we find that $C>0.049$, whence the number of representations of an odd natural number $n$ as the sum of 21 fifth powers of prime numbers is at least as large as

$$
R\left(n, \mathfrak{X}_{0}\right) \gg \mathfrak{S}(n) J(n) .
$$

But by the second conclusion of Lemma 7.2, it follows that $R\left(n, \mathfrak{X}_{0}\right)>0$ for sufficiently large odd natural numbers $n$. Thus the proof of Theorem 2 will be completed on establishing Lemma 7.3.

\section{A singular series deriving from the sieve}

In order to apply the linear sieve to establish Lemma 7.3, we must discuss the consequences of the presence of sifting variables in the representation problem central to our argument. In particular, we must discuss the relevant singular series in some detail. We begin by introducing the notation necessary for the later discussion.

First recall the definition of $S^{*}(q, a)$ from (7.7), and write $S(q, a)$ for $S_{5}(q, a)$, where $S_{5}(q, a)$ is defined as in (2.1). We then define

$$
A_{d}(q, n)=\sum_{\substack{a=1 \\(a, q)=1}}^{q}\left(q^{-1} S\left(q, a d^{5}\right)\right)\left(\varphi(q)^{-1} S^{*}(q, a)\right)^{20} e(-a n / q)
$$

and

$$
\widetilde{\Xi}_{d}(n)=\sum_{q=1}^{\infty} A_{d}(q, n)
$$

On combining the estimate (7.20) with the trivial estimate $\left|S\left(q, a d^{5}\right)\right| \leqslant q$, we find that

$$
A_{d}(q, n) \ll q^{\varepsilon-9},
$$

and hence we deduce that the singular series $\mathfrak{S}_{d}(n)$ converges absolutely. Next define

$$
B_{d}(p, n)=\sum_{l=0}^{\infty} A_{d}\left(p^{l}, n\right)
$$

Also, we define $\gamma=\gamma(p)$ by

$$
\gamma(p)= \begin{cases}2, & \text { when } p=5 \\ 1, & \text { when } p \neq 5,\end{cases}
$$

in accordance with the definition (1.1). Then by Lemma 8.3 of Hua [8], whenever 
$(a, p)=1$ and $l>\gamma(p)$, one has $S^{*}\left(p^{l}, a\right)=0$, and hence

$$
B_{d}(p, n)=\sum_{l=0}^{\gamma} A_{d}\left(p^{l}, n\right)
$$

Further, by (8.3) one has

$$
B_{d}(p, n)=1+O\left(p^{-8}\right),
$$

and hence the infinite product $\prod_{p} B_{d}(p, n)$ is absolutely convergent. A multiplicative change of variables, moreover, reveals that $B_{d}(p, n)$ is equal to either $B_{p}(p, n)$ or $B_{1}(p, n)$, according to whether $p \mid d$ or $p \nmid d$. Furthermore, on referring to Lemma 8.1 of [8] and Lemmata 2.10 and 2.11 of [19], it is apparent that $A_{d}(q, n)$ is a multiplicative function of $q$, and thus we deduce that

$$
\Im_{d}(n)=\prod_{p} B_{d}(p, n)=\left(\prod_{p \nmid d} B_{1}(p, n)\right)\left(\prod_{p \mid d} B_{p}(p, n)\right) .
$$

Next, denote by $M(n, q, s)$ the number of solutions of the congruence

$$
n \equiv x_{1}^{5}+x_{2}^{5}+\ldots+x_{s}^{5} \quad(\bmod q)
$$

with $1 \leqslant x_{j} \leqslant q$ and $\left(x_{j}, q\right)=1 \quad(1 \leqslant j \leqslant s)$. Also, denote by $N(n, q, s)$ the corresponding number of solutions subject to the weaker constraint $\left(x_{j}, q\right)=1$ $(2 \leqslant j \leqslant s)$. Thus we have, in particular,

$$
\begin{aligned}
N\left(n, p^{\gamma}, 21\right) & =\sum_{x=1}^{p^{\gamma}} M\left(n-x^{5}, p^{\gamma}, 20\right) \\
& =\sum_{\substack{x=1 \\
(x, p)=1}}^{p^{\gamma}} M\left(n-x^{5}, p^{\gamma}, 20\right)+\sum_{y=1}^{p^{\gamma-1}} M\left(n-(p y)^{5}, p^{\gamma}, 20\right) \\
& =M\left(n, p^{\gamma}, 21\right)+p^{\gamma-1} M\left(n, p^{\gamma}, 20\right) .
\end{aligned}
$$

But as in Lemma 8.6 of [8] and Lemma 2.12 of [19], one has

$$
\begin{aligned}
B_{1}(p, n) & =\sum_{a=1}^{p^{\gamma}}\left(p^{-\gamma} S\left(p^{\gamma}, a\right)\right)\left(\varphi\left(p^{\gamma}\right)^{-1} S^{*}\left(p^{\gamma}, a\right)\right)^{20} e\left(-a n / p^{\gamma}\right) \\
& =\varphi\left(p^{\gamma}\right)^{-20} N\left(n, p^{\gamma}, 21\right),
\end{aligned}
$$

and

$$
\begin{aligned}
B_{p}(p, n) & =\sum_{a=1}^{p^{\gamma}}\left(\varphi\left(p^{\gamma}\right)^{-1} S^{*}\left(p^{\gamma}, a\right)\right)^{20} e\left(-a n / p^{\gamma}\right) \\
& =p^{\gamma} \varphi\left(p^{\gamma}\right)^{-20} M\left(n, p^{\gamma}, 20\right) .
\end{aligned}
$$

But Lemmata 8.8 and 8.9 of $[\mathbf{8}]$ assert that for all primes $p$, one has $M\left(m, p^{\gamma}, 20\right)>0$ for each even integer $m$. Thus, for all primes $p$, one has $N\left(n, p^{\gamma}, 21\right)>0$ for each integer $n$. On recalling (8.5), (8.6) and (8.9), we therefore arrive at the lower bound

$$
\Im_{1}(n) \gg 1
$$


In view of the lower bound (8.11), it makes sense to define the function

$$
\omega_{n}(d)=\mathfrak{\subseteq}_{d}(n) / \mathfrak{S}_{1}(n) .
$$

We now aim to confirm that $\omega_{n}(d)$ satisfies the conditions relevant to the application of the linear sieve.

Lemma 8.1. The function $\omega_{n}(d)$ is a multiplicative function of $d$, and satisfies

$$
\omega_{n}(p)=1+O\left(p^{-8}\right) \text { and } \omega_{n}\left(p^{l}\right)=\omega_{n}(p),
$$

for all primes $p$ and natural numbers $l$. When $n$ is odd, moreover, we have $0 \leqslant \omega_{n}(p)<p$ for all prime numbers $p$.

Proof. It follows from (8.6) and (8.12) that

$$
\omega_{n}(d)=\prod_{p \mid d}\left(B_{p}(p, n) / B_{1}(p, n)\right) .
$$

Thus $\omega_{n}(d)$ is plainly a multiplicative function of $d$, and on recalling (8.5) it is also immediate that $\omega_{n}(p)=1+O\left(p^{-8}\right)$. Furthermore, the formula (8.13) shows that for each prime $p$ and natural number $l$,

$$
\omega_{n}\left(p^{l}\right)=\frac{B_{p}(p, n)}{B_{1}(p, n)}=\omega_{n}(p) .
$$

It remains only to establish the final assertion of the lemma, and for this we substitute from (8.9) and (8.10) into (8.13) to obtain

$$
\omega_{n}(p)=\frac{p^{\gamma} M\left(n, p^{\gamma}, 20\right)}{N\left(n, p^{\gamma}, 21\right)} .
$$

Consequently, on recalling (8.8), we deduce that

$$
\omega_{n}(p)=\frac{p^{\gamma} M\left(n, p^{\gamma}, 20\right)}{M\left(n, p^{\gamma}, 21\right)+p^{\gamma-1} M\left(n, p^{\gamma}, 20\right)} .
$$

But $M\left(n, p^{\gamma}, 21\right)>0$ for all prime numbers $p$ when $n$ is odd, as a consequence of Lemmata 8.8 and 8.9 of $[\mathbf{8}]$, and hence we obtain the desired bound $0 \leqslant \omega_{n}(p)<p$.

We conclude this section by investigating the relationship between the singular series $\mathfrak{S}(n)$ defined in (7.21), and the singular series $\mathfrak{S}_{1}(n)$ defined in (8.2).

Lemma 8.2. When $z$ is a real number with $z \geqslant 2$, define

$$
W_{n}(z)=\prod_{p<z}\left(1-\omega_{n}(p) / p\right)
$$

Then one has

$$
\Xi_{1}(n) W_{n}(z)=\Im_{(n)} \frac{e^{-\gamma_{0}}}{\log z}\left(1+O\left((\log z)^{-1}\right)\right),
$$

where $\gamma_{0}$ denotes Euler's constant.

Proof. Write

$$
A(q, n)=\sum_{\substack{a=1 \\(a, q)=1}}^{q}\left(\varphi(q)^{-1} S^{*}(q, a)\right)^{21} e(-a n / q)
$$


and

$$
B(p, n)=\sum_{l=0}^{\gamma} A\left(p^{l}, n\right),
$$

and note that the definition (7.21) is equivalent to

$$
\Im(n)=\sum_{q=1}^{\infty} A(q, n) \text {. }
$$

Then by an argument similar to that leading to (8.6), we have

$$
\Im(n)=\prod_{p} B(p, n),
$$

and further,

$$
B(p, n)=p^{\gamma} \varphi\left(p^{\gamma}\right)^{-21} M\left(n, p^{\gamma}, 21\right) .
$$

But by (8.8) and (8.14), we have

$$
1-\frac{\omega_{n}(p)}{p}=\frac{M\left(n, p^{\gamma}, 21\right)}{M\left(n, p^{\gamma}, 21\right)+p^{\gamma-1} M\left(n, p^{\gamma}, 20\right)}=\frac{M\left(n, p^{\gamma}, 21\right)}{N\left(n, p^{\gamma}, 21\right)} .
$$

Thus we deduce from (8.9) and (8.17) that

$$
\begin{aligned}
B_{1}(p, n) & =B(p, n)\left(1-\frac{1}{p}\right) \frac{N\left(n, p^{\gamma}, 21\right)}{M\left(n, p^{\gamma}, 21\right)} \\
& =B(p, n)\left(1-\frac{1}{p}\right)\left(1-\frac{\omega_{n}(p)}{p}\right)^{-1},
\end{aligned}
$$

so that in view of the Euler products (8.6) and (8.16), we have

$$
\varsigma_{1}(n) W_{n}(z)=\Xi(n)\left(\prod_{p<z}\left(1-\frac{1}{p}\right)\right)\left(\prod_{p \geqslant z}\left(1-\frac{1}{p}\right)\left(1-\frac{\omega_{n}(p)}{p}\right)^{-1}\right) .
$$

However, Lemma 8.1 shows that

$$
\prod_{p \geqslant z}\left(1-\frac{1}{p}\right)\left(1-\frac{\omega_{n}(p)}{p}\right)^{-1}=\prod_{p \geqslant z}\left(1+O\left(p^{-9}\right)\right)=1+O\left(z^{-8}\right),
$$

and by Merten's formula one has

$$
\prod_{p<z}\left(1-\frac{1}{p}\right)=e^{-\gamma_{0}}(\log z)^{-1}\left(1+O\left((\log z)^{-1}\right)\right) .
$$

The proof of the lemma is completed by substituting (8.19) and (8.20) into (8.18).

\section{Application of Iwaniec's linear sieve}

We now bring all of our forces to bear on the problem of applying Iwaniec's linear sieve to establish Lemma 7.3. We appeal to the linear sieve in the following form.

\section{Lemma 9.1. Define the functions}

$$
\phi_{0}(u)=\frac{2 e^{\gamma_{0}}}{u} \log (u-1) \quad \text { and } \quad \phi_{1}(u)=\frac{2 e^{\gamma_{0}}}{u},
$$


for $2 \leqslant u \leqslant 3$. Suppose that $\omega(d)$ is a multiplicative function of $d$ satisfying the conditions

$$
0 \leqslant \omega(p)<p \quad \text { and } \quad \omega\left(p^{l}\right)=1+O\left(p^{-1}\right),
$$

for each prime number $p$ and natural number $l$. Let $X$ be a real number with $X \geqslant 3$, let $r(x)$ be a non-negative arithmetical function, and write

$$
E_{d}=\sum_{\substack{P<x<2 P \\ x \equiv 0(\bmod d)}} r(x)-\frac{\omega(d)}{d} X
$$

Let $z, U$ and $V$ be positive real parameters satisfying the inequality

$$
2 \leqslant \frac{\log (U V)}{\log z} \leqslant 3 .
$$

Suppose further that for any sequences $\left(a_{m}\right)$ and $\left(b_{k}\right)$ with

$$
\left|a_{m}\right| \leqslant 1 \text { and }\left|b_{k}\right| \leqslant 1 \text {, }
$$

one has

$$
\sum_{1 \leqslant m \leqslant U} a_{m} \sum_{1 \leqslant k \leqslant V} b_{k} E_{m k} \ll X(\log X)^{-2} .
$$

Then, on writing

$$
W(z)=\prod_{p<z}(1-\omega(p) / p),
$$

one has the lower bound

$$
\sum_{\substack{P<x<2 P \\(x, \Pi(z))=1}} r(x)>X W(z)\left(\phi_{0}\left(\frac{\log (U V)}{\log z}\right)+O\left((\log \log X)^{-1 / 50}\right)\right),
$$

and also the upper bound

$$
\sum_{\substack{P<x<2 P \\(x, \Pi(z))=1}} r(x)<X W(z)\left(\phi_{1}\left(\frac{\log (U V)}{\log z}\right)+O\left((\log \log X)^{-1 / 50}\right)\right) .
$$

Proof. On following the argument of the proof of Theorem 1 of Iwaniec [9], one finds that the introduction of the non-negative weights $r(x)$ is easily accommodated within the latter theorem, and the conclusion claimed in Lemma 9.1 thus follows with little additional effort.

Our initial strategy is to apply Iwaniec's linear sieve to estimate $R\left(n, \mathfrak{X}_{1}\right)$. In order to achieve this objective, we introduce the set of integers

$$
\mathfrak{Y}_{d}=\{P<x<2 P: x \equiv 0(\bmod d)\},
$$

and investigate $R\left(n, \mathfrak{Y}_{d}\right)$ (here and in what follows, we retain the notation of $\S \S 6-8)$. With this end in mind, we define

$$
f_{d}(\alpha)=\sum_{P / d<y<2 P / d} e\left((d y)^{5} \alpha\right)
$$


and when $\mathfrak{B} \subseteq[0,1)$, we write

$$
R\left(n, \mathfrak{Y}_{d} ; \mathfrak{B}\right)=\int_{\mathfrak{B}} f_{d}(\alpha) g_{1}(\alpha)^{2} \mathscr{G}_{2}(\alpha)^{2} e(-n \alpha) d \alpha .
$$

Then by orthogonality we have

$$
R\left(n, \mathfrak{Y}_{d}\right)=R\left(n, \mathfrak{Y}_{d} ;[0,1)\right)=R\left(n, \mathfrak{Y}_{d} ; \mathfrak{N}\right)+R\left(n, \mathfrak{Y}_{d} ; \mathfrak{n}\right)
$$

Recalling (2.1), we abbreviate $S_{5}(q, a)$ to $S(q, a)$, and define also

$$
v(\beta)=\int_{P}^{2 P} e\left(\beta t^{5}\right) d t .
$$

Then by Theorem 4.1 of Vaughan [19], whenever $\alpha \in \mathfrak{N}(q, a) \subseteq \mathfrak{N}$ and $1 \leqslant d \leqslant P L^{-3}$, we have

$$
f_{d}(\alpha)=q^{-1} S\left(q, a d^{5}\right) \int_{P / d}^{2 P / d} e\left((\alpha-a / q) d^{5} t^{5}\right) d t+O(L),
$$

so that by a change of variable we obtain

$$
f_{d}(\alpha)=(d q)^{-1} S\left(q, a d^{5}\right) v(\alpha-a / q)+O(L) .
$$

Thus, since the measure of $\mathfrak{N}$ is $O\left(L^{3} P^{-5}\right)$, we deduce from (7.8) and (9.5) that for $1 \leqslant d \leqslant P L^{-6}$, one has

$$
R\left(n, \mathfrak{Y}_{d} ; \mathfrak{N}\right)=d^{-1} \Im_{d}(n ; L) J_{2}\left(n ; L P^{-5}\right)+O\left(d^{-1} \mathscr{G}_{2}(0)^{2} P^{-2} L^{-2}\right),
$$

where

$$
\Xi_{d}(n ; L)=\sum_{1 \leqslant q \leqslant L} A_{d}(q, n),
$$

with $A_{d}(q, n)$ defined by (8.1), and

$$
J_{2}(n ; W)=\int_{-W}^{W} v(\beta) u_{1}(\beta)^{2} \mathscr{U}_{2}(\beta)^{2} e(-n \beta) d \beta .
$$

Our next step is to complete the singular series and singular integral. First, on recalling (8.2) and (8.3), we find from (9.7) that

$$
\Im_{d}(n)-\Im_{d}(n ; L) \ll \sum_{q>L} q^{\varepsilon-9} \ll L^{-7} .
$$

Next we observe that by (9.4) and (7.7) one has

$$
v(\beta)=\int_{P}^{2 P} \frac{\log P+O(1)}{\log t} e\left(\beta t^{5}\right) d t=u_{1}(\beta) \log P+O\left(P(\log P)^{-1}\right) .
$$

Consequently, by applying trivial estimates in combination with (7.25), we deduce from (7.24) and (9.8) that

$$
\begin{aligned}
J_{2}\left(n ; L P^{-5}\right)-J(n) \log P \ll & P^{3}(\log P)^{-2} \mathscr{U}_{2}(0)^{2} \int_{L P^{-5}}^{\infty}\left(1+P^{5} \beta\right)^{-2} d \beta \\
& +P^{3}(\log P)^{-3} \mathscr{U}_{2}(0)^{2} \int_{0}^{\infty}\left(1+P^{5} \beta\right)^{-2} d \beta \\
\ll & P^{-2}(\log P)^{-3} \mathscr{U}_{2}(0)^{2} .
\end{aligned}
$$


In view of (7.29), therefore, we may conclude that

$$
J_{2}\left(n ; L P^{-5}\right)=J(n)(\log P+O(1)) .
$$

We now turn our attention to the bilinear expressions occurring in the sieve. Let

$$
U=P^{1 / 2-c} \text { and } V=P^{1-32 c},
$$

in which $c=0.0183$, and write

$$
E_{d}=R\left(n, \mathfrak{Y}_{d}\right)-d^{-1} \Im_{d}(n) J_{2}\left(n ; L P^{-5}\right) .
$$

On recalling (8.12), we find that this definition is equivalent to

$$
E_{d}=R\left(n, \mathfrak{Y}_{d}\right)-\frac{\omega_{n}(d)}{d} \Im_{1}(n) J_{2}\left(n ; L P^{-5}\right) .
$$

Also, by (9.3), (9.6), (9.9) together with (9.11) and (7.29), we have

$$
E_{d}=R\left(n, \mathfrak{Y}_{d} ; \mathfrak{n}\right)+O\left(d^{-1} \mathscr{G}_{2}(0)^{2} P^{-2} L^{-2}\right) .
$$

Thus we deduce that for any sequences $\left(a_{m}\right)$ and $\left(b_{k}\right)$ satisfying (9.2), one has

$$
\begin{aligned}
\sum_{1 \leqslant m \leqslant U} a_{m} & \sum_{1 \leqslant k \leqslant V} b_{k} E_{m k} \\
= & \int_{\mathfrak{n}} H(\alpha) g_{1}(\alpha)^{2} \mathscr{G}_{2}(\alpha)^{2} e(-n \alpha) d \alpha+O\left(\mathscr{G}_{2}(0)^{2} P^{-2} L^{-1}\right),
\end{aligned}
$$

where $H(\alpha)=H(\alpha ; \mathbf{a}, \mathbf{b})$ is the exponential sum defined by

$$
H(\alpha)=\sum_{1 \leqslant m \leqslant U} a_{m} \sum_{1 \leqslant k \leqslant V} b_{k} f_{m k}(\alpha) .
$$

Suppose that $\alpha$ is a real number, and suppose also that $a \in \mathbb{Z}$ and $q \in \mathbb{N}$ satisfy the conditions (6.26). We estimate the exponential sum $H(\alpha)$ by dividing up the summations in order to apply Lemmata 3.1 and 3.2 . We put $V_{0}=P^{1 / 2-15 c}$, and observe that

$$
U^{7 / 8} V_{0}^{15 / 8}<P^{7 / 8} \text { and } U^{15 / 8} V_{0}^{-1 / 8}=P^{7 / 8} .
$$

Thus we may apply Lemma 3.2 with $c_{l}=1$ to obtain the estimate

By writing

$$
\begin{aligned}
\sum_{1 \leqslant m \leqslant U} a_{m} & \sum_{1 \leqslant k \leqslant V_{0}} b_{k} f_{m k}(\alpha) \\
& \ll P^{1-1 / 16+\varepsilon}\left(U V_{0}\right)^{1 / 16}+\frac{q^{\varepsilon} w_{5}(q) P(\log P)^{4}}{1+P^{5}|\alpha-a / q|} .
\end{aligned}
$$

$$
\begin{aligned}
\sum_{1 \leqslant m \leqslant U} a_{m} & \sum_{V_{0}<k \leqslant V} b_{k} f_{m k}(\alpha) \\
= & \sum_{P / V<l<2 P / V_{0}}\left(\sum_{\substack{m \mid l \\
1 \leqslant m \leqslant U}} a_{m}\right) \sum_{\substack{P / l<k<2 P / l \\
V_{0}<k \leqslant V}} b_{k} e\left((k l)^{5} \alpha\right),
\end{aligned}
$$


and noting that $P / V>P^{1 / 2}$, we find that a dyadic dissection argument in combination with Lemma 3.1 reveals the bilinear sum on the left-hand side to be

$$
\ll(\log P) \sup _{P / V \leqslant M \leqslant P / V_{0}}\left(P M^{\varepsilon-1 / 32}+(P M)^{1 / 2+\varepsilon}+\frac{q^{\varepsilon} w_{5}(q)^{1 / 2} P(\log P)^{4}}{\left(1+P^{5}|\alpha-a / q|\right)^{1 / 2}}\right) .
$$

Combining the conclusions of (9.15) and (9.16), we find that

$$
H(\alpha) \ll P^{1-c+\varepsilon}+\frac{q^{\varepsilon} w_{5}(q)^{1 / 2} P(\log P)^{5}}{\left(1+P^{5}|\alpha-a / q|\right)^{1 / 2}} .
$$

We now apply Lemma 6.3, which in view of (9.17) yields the estimate

$$
\int_{\mathfrak{r}} H(\alpha) g_{1}(\alpha)^{2} \mathscr{G}_{2}(\alpha)^{2} e(-n \alpha) d \alpha \ll \mathscr{G}_{2}(0)^{2} P^{-2}(\log P)^{-100},
$$

whence by (9.13), for any sequences $\left(a_{m}\right)$ and $\left(b_{k}\right)$ satisfying (9.2),

$$
\sum_{1 \leqslant m \leqslant U} a_{m} \sum_{1 \leqslant k \leqslant V} b_{k} E_{m k} \ll \mathscr{G}_{2}(0)^{2} P^{-2}(\log P)^{-100} \text {. }
$$

On recalling the estimates (8.11), (7.29) and (9.11), we therefore conclude that

$$
\sum_{1 \leqslant m \leqslant U} a_{m} \sum_{1 \leqslant k \leqslant V} b_{k} E_{m k} \ll \Im_{1}(n) J_{2}\left(n ; L P^{-5}\right)(\log P)^{-98} .
$$

We are finally equipped to establish the lower bound for $R\left(n, \mathfrak{X}_{1}\right)$ presented in Lemma 7.3. With $n$ a fixed odd natural number, we take the arithmetical function $r(x)$ equal to the number of solutions of the equation (7.1) with $p_{l}$ satisfying the conditions (7.2) for $1 \leqslant l \leqslant 20$. On recalling (7.3), we have $z=(2 P)^{1 / 3}$, and so a smidgen of computation verifies that indeed

so that on taking

$$
2<\frac{\log (U V)}{\log z}<3
$$

$$
X=\Im_{1}(n) J_{2}\left(n ; L P^{-5}\right),
$$

we find that the hypotheses necessary for the application of Lemma 9.1 are satisfied, by virtue of (9.18) and Lemma 8.1. Consequently, employing a transparent modification of the notation of the statement of Lemma 9.1, we arrive at the lower bound

$$
R\left(n, \mathfrak{X}_{1}\right)>\mathfrak{S}_{1}(n) J_{2}\left(n ; L P^{-5}\right) W_{n}(z)\left(\phi_{0}\left(\frac{\log (U V)}{\log z}\right)+O\left((\log \log P)^{-1 / 50}\right)\right) .
$$

Further, with an application of Lemma 8.2 together with (9.1), (9.11) and (9.12), one obtains

$$
R\left(n, \mathfrak{X}_{1}\right)>\subseteq(n) J(n)\left(\frac{\log P}{\log z}\right)\left(\frac{2}{\kappa} \log (\kappa-1)+O\left((\log \log P)^{-1 / 50}\right)\right),
$$

where

$$
\kappa=\frac{\log (U V)}{\log z}=3\left(\frac{3}{2}-33 c\right)+O\left((\log P)^{-1}\right) .
$$

The first conclusion of Lemma 7.3 is therefore immediate from (9.19). 
We now turn our hand against the proof of the upper bound for $R\left(n, \mathfrak{X}_{3}\right)$ presented in Lemma 7.3. It is here that we apply the reversal of rôles technique in combination with Lemma 9.1. When $\mathfrak{X} \subseteq(P, 2 P) \cap \mathbb{Z}$, we now denote by $R^{\prime}(n, \mathfrak{X})$ the number of representations of the natural number $n$ in the form

$$
n=x^{5}+y^{5}+p_{2}^{5}+p_{3}^{5}+\ldots+p_{20}^{5},
$$

with $x \in \mathfrak{X}_{3}, y \in \mathfrak{X}$, and with $p_{l}$ satisfying (7.2) for $2 \leqslant l \leqslant 20$. Since $\mathfrak{X}_{0} \subseteq \mathfrak{X}_{1}$, it is apparent that

$$
R\left(n, \mathfrak{X}_{3}\right)=R^{\prime}\left(n, \mathfrak{X}_{0}\right) \leqslant R^{\prime}\left(n, \mathfrak{X}_{1}\right) .
$$

Define

$$
h_{2}(\alpha)=\sum_{x \in \mathfrak{X}_{3}} e\left(\alpha x^{5}\right),
$$

and when $\mathfrak{B} \subseteq[0,1)$, write

$$
R^{\prime}\left(n, \mathfrak{Y}_{d} ; \mathfrak{B}\right)=\int_{\mathfrak{B}} f_{d}(\alpha) h_{2}(\alpha) g_{1}(\alpha) \mathscr{G}_{2}(\alpha)^{2} e(-n \alpha) d \alpha .
$$

By orthogonality, therefore, one has

$$
R^{\prime}\left(n, \mathfrak{Y}_{d}\right)=R^{\prime}\left(n, \mathfrak{Y}_{d} ;[0,1)\right)=R^{\prime}\left(n, \mathfrak{Y}_{d} ; \mathfrak{N}\right)+R^{\prime}\left(n, \mathfrak{Y}_{d} ; \mathfrak{n}\right)
$$

We estimate $h_{2}(\alpha)$ by means of Lemma 7.1, thereby obtaining for $\alpha \in \mathfrak{N}(q, a) \subseteq \mathfrak{N}$ the relation

$$
h_{2}(\alpha)=\varphi(q)^{-1} S^{*}(q, a) u\left(\alpha-a / q ; 1-32 c, \frac{1}{2}\right)+O\left(P L^{-5}\right) .
$$

In a by now familiar fashion (compare the argument leading to (9.6)), the latter estimate, in combination with (9.5), (7.8), (7.20) and (9.9), readily yields for $1 \leqslant d \leqslant P L^{-6}$ the estimate

$$
R^{\prime}\left(n, \mathfrak{Y}_{d} ; \mathfrak{N}\right)=d^{-1} \mathfrak{S}_{d}(n) J_{3}\left(n ; L P^{-5}\right)+O\left(d^{-1} \mathscr{G}_{2}(0)^{2} P^{-2} L^{-2}\right),
$$

where

$$
J_{3}(n ; W)=\int_{-W}^{W} v(\beta) u\left(\beta ; 1-32 c, \frac{1}{2}\right) u_{1}(\beta) \mathscr{U}_{2}(\beta)^{2} e(-n \beta) d \beta .
$$

But by applying trivial estimates in combination with (7.25) and (9.10), we find that

$$
\begin{aligned}
& J_{3}\left(n ; L P^{-5}\right)-(\log P) \int_{-L P^{-5}}^{L P^{-5}} u_{1}(\beta)^{2} u\left(\beta ; 1-32 c, \frac{1}{2}\right) \mathscr{U}_{2}(\beta)^{2} e(-n \beta) d \beta \\
& \ll P^{3}(\log P)^{-3} \mathscr{U}_{2}(0)^{2} \int_{0}^{L P^{-5}}\left(1+P^{5} \beta\right)^{-1} d \beta \\
& \ll P^{-2}(\log P)^{-3} \mathscr{U}_{2}(0)^{2} \log L .
\end{aligned}
$$

Then by exploiting (7.24), (7.25), (7.29), (9.10) and Lemma 7.1, we deduce by an argument paralleling the deduction of (7.28) from (7.18) that

$$
\begin{aligned}
J_{3}\left(n ; L P^{-5}\right)= & J(n)\left(\log \left(\frac{32 c}{1-32 c}\right) \log P+O(\log L)\right) \\
& +O\left(\mathscr{G}_{2}(0)^{2} P^{-2}(\log P)^{-3}\right) \\
= & J(n)\left(\log \left(\frac{32 c}{1-32 c}\right) \log P+O(\log L)\right) .
\end{aligned}
$$


We now put

$$
E_{d}^{\prime}=R^{\prime}\left(n, \mathfrak{Y}_{d}\right)-d^{-1} \mathfrak{\subseteq}_{d}(n) J_{3}\left(n ; L P^{-5}\right),
$$

so that in view of (8.12), we have

$$
E_{d}^{\prime}=R^{\prime}\left(n, \mathfrak{Y}_{d}\right)-\frac{\omega_{n}(d)}{d} \Im_{1}(n) J_{3}\left(n ; L P^{-5}\right) .
$$

Then by (9.23) and (9.24), for any sequences $\left(a_{m}\right)$ and $\left(b_{k}\right)$ satisfying (9.2), it follows from (9.14) that

$$
\begin{aligned}
\sum_{1 \leqslant m \leqslant U} a_{m} \sum_{1 \leqslant k \leqslant V} b_{k} E_{m k}^{\prime}= & \int_{\mathfrak{n}} H(\alpha) h_{2}(\alpha) g_{1}(\alpha) \mathscr{G}_{2}(\alpha)^{2} e(-n \alpha) d \alpha \\
& +O\left(\mathscr{G}_{2}(0)^{2} P^{-2} L^{-1}\right) .
\end{aligned}
$$

Then on recalling (9.17), we may apply Lemma 6.3 to conclude that under the same conditions,

$$
\sum_{1 \leqslant m \leqslant U} a_{m} \sum_{1 \leqslant k \leqslant V} b_{k} E_{m k}^{\prime} \ll \mathscr{G}_{2}(0)^{2} P^{-2}(\log P)^{-100},
$$

whence by (8.11), (7.29) and (9.25),

$$
\sum_{1 \leqslant m \leqslant U} a_{m} \sum_{1 \leqslant k \leqslant V} b_{k} E_{m k}^{\prime} \ll \Im_{1}(n) J_{3}\left(n ; L P^{-5}\right)(\log P)^{-98} .
$$

We are finally again equipped for our application of Lemma 9.1. With $n$ a fixed odd natural number, we take the arithmetical function $r(y)$ equal to the number of solutions of the equation (9.21) with $x \in \mathfrak{X}_{3}$ and $p_{l}$ satisfying the conditions (7.2) for $2 \leqslant l \leqslant 20$. As in our previous application of Lemma 9.1, we find that on taking

$$
X=\Im_{1}(n) J_{3}\left(n ; L P^{-5}\right),
$$

the hypotheses necessary for the application of Lemma 9.1 are satisfied, on account of the upper bound (9.26) together with Lemma 8.1. Consequently, employing a natural modification of the notation of the statement of Lemma 9.1, we grasp the upper bound

$$
R^{\prime}\left(n, \mathfrak{X}_{1}\right)<\Xi_{1}(n) J_{3}\left(n ; L P^{-5}\right) W_{n}(z)\left(\phi_{1}\left(\frac{\log (U V)}{\log z}\right)+O\left((\log \log P)^{-1 / 50}\right)\right) .
$$

Thus an application of Lemma 8.2 in combination with (9.1), (9.12), (9.25) and (9.22) yields

$$
R\left(n, \mathfrak{X}_{3}\right)<\subseteq(n) J(n)\left(\frac{\log P}{\log z}\right) \log \left(\frac{32 c}{1-32 c}\right)\left(\frac{2}{\kappa}+O\left((\log \log P)^{-1 / 50}\right)\right),
$$

where $\kappa$ is the number defined in (9.20). The second conclusion of Lemma 7.3 follows immediately.

Given the discussion concluding $\S 7$, the proof of Theorem 2 is at last complete.

\section{References}

1. J. BRÜDERN, 'Sieves, the circle method, and Waring's problem for cubes' (Habilitationschrift, Göttingen 1991), Mathematica Gottingensis 51 (1991). 
2. J. BRÜDERN, 'A sieve approach to the Waring-Goldbach problem, I: sums of four cubes', Ann. Sci. École Norm. Sup. (4) 28 (1995) 461-476.

3. J. BRÜDERN, 'A sieve approach to the Waring-Goldbach problem, II. On the seven cubes theorem', Acta Arith. 72 (1995) 211-227.

4. H. Davenport, 'On Waring's problem for fourth powers', Ann. of Math. 40 (1939) 731-747.

5. D. R. Heath-Brown, 'Three primes and an almost-prime in arithmetic progression', J. London Math. Soc. (2) 23 (1981) 396-414.

6. L.-K. HuA, 'On the representation of numbers as the sums of the powers of primes', Math. Z. 44 (1938) 335-346.

7. L.-K. HuA, Abschätzungen von Exponentialsummen und ihre Anwendung in der Zahlentheorie, Enzyklopädie der Mathematischen Wissenschaften, Band I 2, Heft 13, Teil 1 (Teubner, Leipzig, 1959).

8. L.-K. HuA, Additive theory of prime numbers (American Mathematical Society, Providence, RI, 1965).

9. H. Iwaniec, 'A new form of the error term in the linear sieve', Acta Arith. 37 (1980) 307-320.

10. K. KaWADA, 'Note on the sum of cubes of primes and an almost prime', Arch. Math. (Basel) 69 (1997) 13-19.

11. K. Thanigasalam, 'Improvement on Davenport's iterative method and new results in additive number theory, I', Acta Arith. 46 (1985) 1-31.

12. K. Thanigasalam, 'Improvement on Davenport's iterative method and new results in additive number theory, II. Proof that $G(5) \leqslant 22$ ', Acta Arith. 46 (1986) 91-112.

13. K. Thanigasalam, 'Improvement on Davenport's iterative method and new results in additive number theory, III', Acta Arith. 48 (1987) 97-116.

14. K. Thanigasalam, 'On sums of positive integral powers and simple proof of $G(6) \leqslant 31$ ', Bull. Calcutta Math. Soc. 81 (1989) 279-294.

15. K. Thanigasalam, 'On admissible exponents for $k$ th powers', Bull. Calcutta Math. Soc. 86 (1994) 175-178.

16. R. C. Vaughan, 'Recent work in additive prime number theory', Proceedings of the International Congress of Mathematicians, Helsinki, 1978 (Acad. Sci. Fennica, Helsinki, 1980) 389-394.

17. R. C. Vaughan, 'On Waring's problem for smaller exponents', Proc. London Math. Soc. (3) 52 (1986) 445-463.

18. R. C. Vaughan, 'A new iterative method in Waring's problem', Acta Math. 162 (1989) 1-71.

19. R. C. Vaughan, The Hardy-Littlewood method, 2nd edn (Cambridge University Press, 1997).

20. I. M. Vinogradov, 'Representation of an odd number as a sum of three primes', C. R. Acad. Sci. URSS 15 (1937) 6-7.

21. I. M. Vinogradov, 'Some theorems concerning the theory of primes', Rec. Math. 2 (1937) $179-195$

Koichi Kawada

Department of Mathematics

Faculty of Education

Iwate University

Morioka

020-8550 Japan

kawada@iwate-u.ac.jp
Trevor D. Wooley

Department of Mathematics

University of Michigan

East Hall

525 East University Avenue

Ann Arbor

MI 48109-1109

USA

wooley@math.lsa.umich.edu 